\title{
Tevatron Run II combination of the effective leptonic electroweak mixing angle
}

T. Aaltonen, ${ }^{21, \dagger}$ V. M. Abazov, ${ }^{13, \$}$ B. Abbott, ${ }^{11, *}$ B. S. Acharya, ${ }^{79, \$}$ M. Adams,${ }^{97, \sharp}$ T. Adams, ${ }^{96, \$}$ J. P. Agnew, ${ }^{93, \$}$ G. D. Alexeev, ${ }^{13, \sharp}$ G. Alkhazov, ${ }^{87, \$}$ A. Alton, ${ }^{31, k k, \sharp}$ S. Amerio, ${ }^{39 a, 39 b, \dagger}$ D. Amidei, ${ }^{31, \dagger}$ A. Anastassov, ${ }^{15, w, \uparrow}$ A. Annovi, ${ }^{17, \uparrow}$ J. Antos, ${ }^{12, \dagger}$ G. Apollinari, ${ }^{15, \dagger}$ J. A. Appel, ${ }^{15, \dagger}$ T. Arisawa, ${ }^{51, \dagger}$ A. Artikov, ${ }^{1, \dagger}$ J. Asaadi, ${ }^{47, \dagger}$ W. Ashmanskas, ${ }^{15, \dagger}$ A. Askew,${ }^{96, *}$

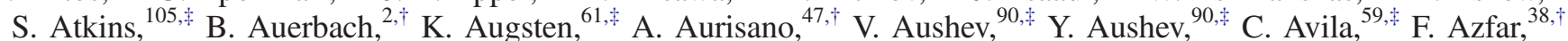
F. Badaud, ${ }^{64, \$}$ W. Badgett,${ }^{15, \dagger}$ T. Bae,${ }^{25, \dagger}$ L. Bagby, ${ }^{15, \$}$ B. Baldin, ${ }^{15, *}$ D. V. Bandurin, ${ }^{12, \$}$ S. Banerjee, ${ }^{79, \sharp}$

A. Barbaro-Galtieri, ${ }^{26, \dagger}$ E. Barberis, ${ }^{106,{ }^{\dagger}}$ P. Baringer, ${ }^{104, \$}$ V. E. Barnes, ${ }^{43, \dagger}$ B. A. Barnett, ${ }^{23, \dagger}$ P. Barria, ${ }^{41 a, 41 c, \dagger}$ J. F. Bartlett,,${ }^{15,}$ P. Bartos, ${ }^{12, \dagger}$ U. Bassler, ${ }^{69, \$}$ M. Bauce, ${ }^{39 a, 39 b, \dagger}$ V. Bazterra, ${ }^{97, \$}$ A. Bean ${ }^{104, \$}$ F. Bedeschi ${ }^{41 a, \dagger}$ M. Begalli, ${ }^{56, \ddagger}$ S. Behari, ${ }^{15, \dagger}$ L. Bellantoni, ${ }^{15, \$}$ G. Bellettini, ${ }^{41 a, 41 b, \uparrow}$ J. Bellinger, ${ }^{53, \dagger}$ D. Benjamin, ${ }^{14, \dagger}$ A. Beretvas, ${ }^{1, \dagger}$ S. B. Beri, ${ }^{77, \sharp}$ G. Bernardi ${ }^{68, *}$

R. Bernhard, ${ }^{73,}$ I. Bertram, ${ }^{91,}$ M. Besançon, ${ }^{69,}$ R. Beuselinck, ${ }^{92, \$}$ P. C. Bhat, ${ }^{15, \$}$ S. Bhatia, ${ }^{107, \$}$ V. Bhatnagar, ${ }^{77, \$}$

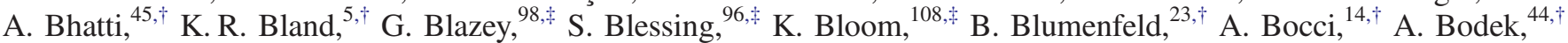
A. Boehnlein, ${ }^{15, *}$ D. Boline ${ }^{112, *}$ E. E. Boos,${ }^{85, \$}$ G. Borissov, ${ }^{91,+}$ D. Bortoletto, ${ }^{43, \dagger}$ M. Borysova,${ }^{90, u u, \sharp}$ J. Boudreau ${ }^{42, \dagger}$ A. Boveia, ${ }^{11, \uparrow}$ A. Brandt, ${ }^{119, \$}$ O. Brandt ${ }^{74,}$ L. Brigliadori, ${ }^{6 a, 6 b, \dagger}$ M. Brochmann, ${ }^{123, \$}$ R. Brock, ${ }^{32, \ddagger}$ C. Bromberg, ${ }^{32, \dagger}$

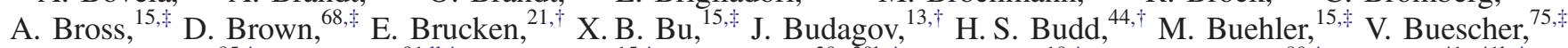

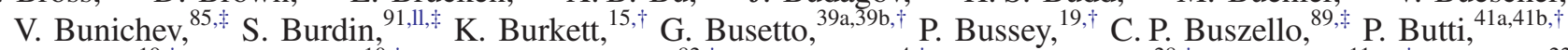
A. Buzatu, ${ }^{19, \dagger}$ A. Calamba ${ }^{10, \dagger}$ E. Camacho-Pérez, ${ }^{82, \uparrow}$ S. Camarda ${ }^{4, \dagger}$ M. Campanelli, ${ }^{28, \dagger}$ F. Canelli, ${ }^{11, e e, \dagger}$ B. Carls, ${ }^{22, \uparrow}$

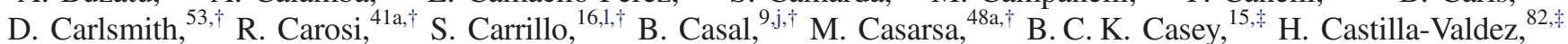
A. Castro, ${ }^{6 a, 6 b, \dagger}$ P. Catastini,${ }^{20, \dagger}$ S. Caughron, ${ }^{32, \uparrow}$ D. Cauz,${ }^{48 a, 48 b, 48 c,{ }^{\prime}}$ V. Cavaliere,${ }^{22, \uparrow}$ A. Cerri, ${ }^{26, e, \dagger}$ L. Cerrito, ${ }^{28, r, \dagger}$ S. Chakrabarti, ${ }^{11, *}$ K. M. Chan, ${ }^{102, \$}$ A. Chandra, ${ }^{121, \$}$ E. Chapon, ${ }^{69, \$}$ G. Chen,${ }^{104, \$}$ Y. C. Chen, ${ }^{1, \dagger}$ M. Chertok, ${ }^{7, \dagger}$ G. Chiarelli, ${ }^{41 a, \dagger}$ G. Chlachidze, ${ }^{15, \dagger}$ K. Cho, ${ }^{25, \dagger}$ S. W. Cho, ${ }^{81,}$ S. Choi, ${ }^{81, \hbar}$ D. Chokheli, ${ }^{13, \dagger}$ B. Choudhary, ${ }^{78,}$ S. Cihangir, ${ }^{15, *, *}$ D. Claes ${ }^{108, \$}$ A. Clark, ${ }^{18, \dagger}$ C. Clarke, ${ }^{52, \dagger}$ J. Clutter ${ }^{104, \$}$ M. E. Convery, ${ }^{15, \dagger}$ J. Conway, ${ }^{7, \dagger}$ M. Cooke, ${ }^{15, t, \neq}$ W. E. Cooper, ${ }^{15, \$}$ M. Corbo, ${ }^{15, z, \dagger}$ M. Corcoran, ${ }^{121, *}$ M. Cordelli, ${ }^{17, \dagger}$ F. Couderc, ${ }^{69,}$ M.-C. Cousinou ${ }^{66, \$}$ C. A. Cox,${ }^{7, \dagger}$ D. J. Cox, ${ }^{7, \dagger}$ M. Cremonesi, ${ }^{41 a, \dagger}$ D. Cruz ${ }^{47, \dagger}$ J. Cuevas, ${ }^{9, y, \dagger}$ R. Culbertson, ${ }^{15, \dagger}$ J. Cuth, ${ }^{75, \$}$ D. Cutts, ${ }^{11,, \$}$ A. Das, ${ }^{120, \$}$ N. d'Ascenzo, ${ }^{15, v, \dagger}$ M. Datta, ${ }^{15, h h, \uparrow}$ G. Davies, ${ }^{92, \$}$ P. de Barbaro, ${ }^{44, \uparrow}$ S. J. de Jong, ${ }^{83,84, \$}$ E. De La Cruz-Burelo, ${ }^{82, \$}$ F. Déliot, ${ }^{69, *}$ R. Demina, ${ }^{44, \$}$ L. Demortier, ${ }^{45, \dagger}$ M. Deninno, ${ }^{6 a, \dagger}$ D. Denisov, ${ }^{15, \$}$ S. P. Denisov, ${ }^{86, \$}$ M. D’Errico, ${ }^{39 a, 39 b, \dagger}$ S. Desai, ${ }^{15, \$}$ C. Deterre, ${ }^{93, m m, \$}$ K. DeVaughan, ${ }^{108, \sharp}$ F. Devoto, ${ }^{21, \dagger}$ A. Di Canto, ${ }^{41 a, 41 b, \dagger}$ B. Di Ruzza,${ }^{15, p, \dagger}$ H. T. Diehl, ${ }^{15,}$ M. Diesburg, ${ }^{15,}$ P. F. Ding ${ }^{93}$

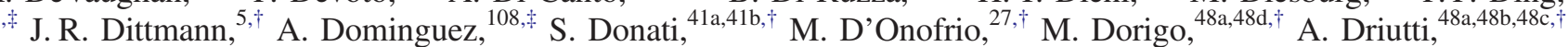
A. Drutskoy, ${ }^{33, z z, \sharp}$ A. Dubey ${ }^{78, \$}$ L. V. Dudko, ${ }^{85, *}$ A. Duperrin,${ }^{66, \$}$ S. Dutt, ${ }^{77, \$}$ M. Eads, ${ }^{98, *}$ K. Ebina,${ }^{51, \uparrow}$ R. Edgar, ${ }^{31, \dagger}$ D. Edmunds, ${ }^{32, \$}$ A. Elagin, ${ }^{1, \dagger}$ J. Ellison, ${ }^{95,}$ V. D. Elvira, ${ }^{15, \$}$ Y. Enari, ${ }^{68, *}$ R. Erbacher ${ }^{7, \dagger}$ S. Errede ${ }^{22, \dagger}$ B. Esham, ${ }^{22, \dagger}$ H. Evans, ${ }^{100, \$}$ A. Evdokimov, ${ }^{97, \$}$ V. N. Evdokimov, ${ }^{86, \$}$ S. Farrington, ${ }^{38, \uparrow}$ A. Fauré, ${ }^{69,}$ L. Feng, ${ }^{98, \$}$ T. Ferbel, ${ }^{44, \$}$ J. P. Fernández Ramos,${ }^{29, \dagger}$ F. Fiedler, ${ }^{75, \$}$ R. Field, ${ }^{16, \dagger}$ F. Filthaut,${ }^{83,84, \$}$ W. Fisher, ${ }^{32, \$}$ H. E. Fisk, ${ }^{15, \$}$ G. Flanagan, ${ }^{15, t, \dagger}$ R. Forrest, ${ }^{7, \dagger}$ M. Fortner, ${ }^{98,}$ H. Fox, ${ }^{91, \dagger}$ J. Franc, ${ }^{61,}$ M. Franklin, ${ }^{20, \dagger}$ J. C. Freeman, ${ }^{15, \dagger}$ H. Frisch, ${ }^{11, \dagger}$ S. Fuess, ${ }^{15, \$}$

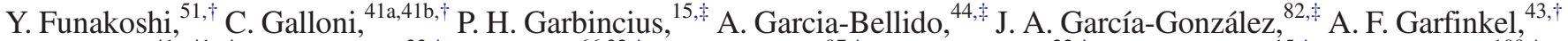
P. Garosi ${ }^{41 \mathrm{a}, 41 \mathrm{c}, \dagger}$ V. Gavrilov, ${ }^{33, \$}$ W. Geng, ${ }^{66,32, \$}$ C. E. Gerber, ${ }^{97, \$}$ H. Gerberich, ${ }^{22, \dagger}$ E. Gerchtein, ${ }^{15, \dagger}$ Y. Gershtein,${ }^{109, \$}$ S. Giagu, ${ }^{46 a, \dagger}$ V. Giakoumopoulou, ${ }^{3, \dagger}$ K. Gibson,${ }^{42, \dagger}$ C. M. Ginsburg, ${ }^{15, \dagger}$ G. Ginther, ${ }^{15, \$}$ N. Giokaris, ${ }^{3, * \dagger}$ P. Giromini, ${ }^{17, \dagger}$ V. Glagolev, ${ }^{13, \dagger}$ D. Glenzinski, ${ }^{15, \dagger}$ O. Gogota, ${ }^{90,+}$ M. Gold ${ }^{34, \uparrow}$ D. Goldin, ${ }^{47, \dagger}$ A. Golossanov, ${ }^{15, \dagger}$ G. Golovanov, ${ }^{13, ;}$ G. Gomez, ${ }^{9, \dagger}$ G. Gomez-Ceballos, ${ }^{30, \uparrow}$ M. Goncharov, ${ }^{30, \uparrow}$ O. González López, ${ }^{29, \dagger}$ I. Gorelov, ${ }^{34, \uparrow}$ A. T. Goshaw, ${ }^{14, \uparrow}$

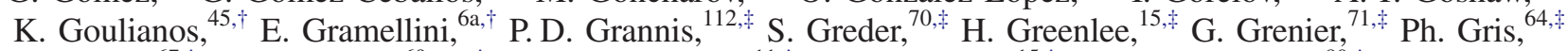
J.-F. Grivaz, ${ }^{67, \ddagger}$ A. Grohsjean, ${ }^{69, m m, *}$ C. Grosso-Pilcher, ${ }^{11, \dagger}$ S. Grünendahl,${ }^{15, \$}$ M. W. Grünewald, ${ }^{80, \$}$ T. Guillemin ${ }^{67, \sharp}$ J. Guimaraes da Costa, ${ }^{20, \uparrow}$ G. Gutierrez, ${ }^{15, \$}$ P. Gutierrez, ${ }^{115, \$}$ S. R. Hahn, ${ }^{15, \dagger}$ J. Haley, ${ }^{116, \$}$ J. Y. Han, ${ }^{44, \dagger}$ L. Han ${ }^{58, \ddagger}$ F. Happacher, ${ }^{17, \dagger}$ K. Hara,${ }^{49, \dagger}$ K. Harder, ${ }^{93,}$ M. Hare,${ }^{50, \dagger}$ A. Harel, ${ }^{44, \uparrow}$ R. F. Harr, ${ }^{52, \uparrow}$ T. Harrington-Taber, ${ }^{15, m, \dagger}$

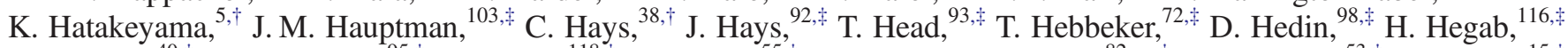
J. Heinrich, ${ }^{40, \dagger}$ A. P. Heinson, ${ }^{95,}$ U. Heintz, ${ }^{118,}$ C. Hensel, ${ }^{55,}$ I. Heredia-De La Cruz ${ }^{82, n n, \sharp}$ M. Herndon, ${ }^{53, \dagger}$ K. Herner, ${ }^{15,}$ G. Hesketh, ${ }^{93, p p, \sharp}$ M. D. Hildreth, ${ }^{102, \$}$ R. Hirosky, ${ }^{122, \$}$ T. Hoang,${ }^{96, \$}$ J. D. Hobbs ${ }^{112, \$}$ A. Hocker, ${ }^{15, \dagger}$ B. Hoeneisen, ${ }^{63, \$}$ J. Hogan ${ }^{121, \$}$ M. Hohlfeld, ${ }^{75, \sharp}$ J. L. Holzbauer, ${ }^{107, \sharp}$ Z. Hong, ${ }^{47, w, \dagger}$ W. Hopkins, ${ }^{15, f, \dagger}$ S. Hou, ${ }^{1, \dagger}$ I. Howley, ${ }^{119, \sharp}$ Z. Hubacek, ${ }^{61,69, \$}$ R. E. Hughes,${ }^{35, \dagger}$ U. Husemann,${ }^{54, \dagger}$ M. Hussein, ${ }^{32, c c, \dagger}$ J. Huston, ${ }^{32, \dagger}$ V. Hynek, ${ }^{61, \$}$ I. Iashvili, ${ }^{111, \sharp}$ Y. Ilchenko, ${ }^{120, \$}$

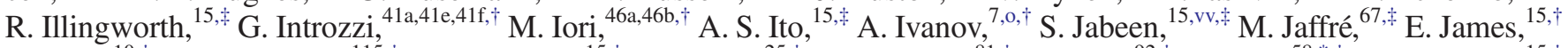
D. Jang, ${ }^{10, \dagger}$ A. Jayasinghe, ${ }^{115, \$}$ B. Jayatilaka, ${ }^{15, \dagger}$ E. J. Jeon, ${ }^{25, \dagger}$ M. S. Jeong, ${ }^{81,}$ R. Jesik, ${ }^{92, \$}$ P. Jiang, ${ }^{58, *,}$ S. Jindariani, ${ }^{15, \dagger}$ K. Johns, ${ }^{94, \sharp}$ E. Johnson, ${ }^{32, \$}$ M. Johnson, ${ }^{15, \$}$ A. Jonckheere, ${ }^{15, \$}$ M. Jones,${ }^{43, \dagger}$ P. Jonsson, ${ }^{92, \$}$ K. K. Joo, ${ }^{25, \dagger}$ J. Joshi, $^{95, \sharp}$

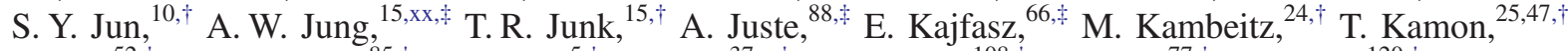

P. E. Karchin, ${ }^{52, \uparrow}$ D. Karmanov, ${ }^{8, \$}$ A. Kasmi, ${ }^{5, \dagger}$ Y. Kato, ${ }^{37, n, \uparrow}$ I. Katsanos, ${ }^{108, \$}$ M. Kaur, ${ }^{77, \$}$ R. Kehoe, ${ }^{120, \$}$ S. Kermiche, ${ }^{66, \$}$ 
W. Ketchum, ${ }^{11, i i, \dagger}$ J. Keung, ${ }^{40, \dagger}$ N. Khalatyan, ${ }^{15}$ A. Khanov, ${ }^{116,}$ A. Kharchilava, ${ }^{11, *}$ Y. N. Kharzheev, ${ }^{13}$, B. Kilminster, ${ }^{15,}$

${ }^{e, \dagger}$ D. H. Kim, ${ }^{25, \dagger}$ H. S. Kim, ${ }^{15, b b, \dagger}$ J. E. Kim, ${ }^{25, \dagger}$ M. J. Kim, ${ }^{17,}$ S. H. Kim, ${ }^{49, \dagger}$ S. B. Kim, ${ }^{25, \dagger}$ Y. J. Kim, ${ }^{25, \dagger}$ Y. K. Kim, ${ }^{1, \dagger}$

N. Kimura ${ }^{51, \dagger}$ M. Kirby, ${ }^{15, \dagger}$ I. Kiselevich, ${ }^{33, \ddagger}$ J. M. Kohli, ${ }^{77, \dagger}$ K. Kondo, ${ }^{51, *, \dagger}$ D. J. Kong, ${ }^{25, \dagger}$ J. Konigsberg, $^{16, \dagger}$

A. V. Kotwal, ${ }^{14, \dagger}$ A. V. Kozelov, ${ }^{86,}$ J. Kraus, ${ }^{107,}$ M. Kreps ${ }^{24, \dagger}{ }^{\dagger}$ J. Kroll, ${ }^{40, \dagger}$ M. Kruse, ${ }^{14, \dagger}$ T. Kuhr, ${ }^{24,}$ A. Kumar, ${ }^{111, \$}$

A. Kupco, ${ }^{62, \ddagger}$ M. Kurata ${ }^{49, \dagger}$ T. Kurča, ${ }^{71,}$ V. A. Kuzmin, ${ }^{85,+}$ A. T. Laasanen, ${ }^{43, \dagger}$ S. Lammel, ${ }^{15, \dagger}$ S. Lammers, ${ }^{100, \$}$

M. Lancaster, ${ }^{28, \dagger}$ K. Lannon, ${ }^{35, x, \dagger}$ G. Latino, ${ }^{41 a, 41 c, \dagger}$ P. Lebrun, ${ }^{71, \ddagger}$ H. S. Lee, ${ }^{81,}$ H. S. Lee, ${ }^{25, \dagger}$ J. S. Lee, ${ }^{25, \dagger}$ S. W. Lee, ${ }^{103, *}$

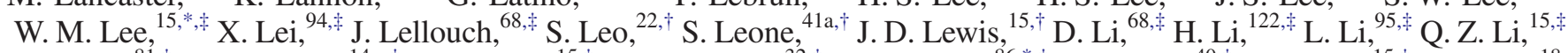
J. K. Lim, ${ }^{81, *}$ A. Limosani, ${ }^{14, s, \dagger}$ D. Lincoln, ${ }^{15, *}$ J. Linnemann, ${ }^{32, *}$ V. V. Lipaev, ${ }^{86, *}$ E. Lipeles, ${ }^{40, \dagger}$ R. Lipton, ${ }^{15,+}$ A. Lister, ${ }^{18,}$ ${ }^{a}, \dagger$ H. Liu, ${ }^{120, \ddagger}$ Q. Liu, ${ }^{43, \dagger}$ T. Liu, ${ }^{15, \dagger}$ Y. Liu, ${ }^{58, \ddagger}$ A. Lobodenko, ${ }^{87, \dagger}$ S. Lockwitz, ${ }^{54, \dagger}$ A. Loginov, ${ }^{54, \dagger}$ M. Lokajicek, ${ }^{62, \dagger}$

R. Lopes de Sa, ${ }^{15,}$ D. Lucchesi, ${ }^{39 a, 39 b, \dagger}$ A. Lucà, ${ }^{17,15, \dagger}$ J. Lueck, ${ }^{24, \dagger}$ P. Lujan, ${ }^{26, \dagger}$ P. Lukens, ${ }^{15, \dagger}$ R. Luna-Garcia, ${ }^{82, q q, 末}$ G. Lungu, ${ }^{4, \dagger}$ A. L. Lyon, ${ }^{15, \$}$ J. Lys,${ }^{26, *, \dagger}$ R. Lysak, ${ }^{12, d, \dagger}$ A. K. A. Maciel,${ }^{55, \ddagger}$ R. Madar, ${ }^{73, \ddagger}$ R. Madrak, ${ }^{15, \dagger}$ P. Maestro, ${ }^{41 a, 41 c, \dagger}$ R. Magaña-Villalba, ${ }^{82, \$}$ S. Malik, ${ }^{45, \dagger}$ S. Malik, ${ }^{108, \ddagger}$ V. L. Malyshev, ${ }^{13,}$ G. Manca, ${ }^{27, b, \dagger}$ A. Manousakis-Katsikakis, ${ }^{3, \dagger}$ J. Mansour, ${ }^{74,}$ L. Marchese,${ }^{6 a, j, \dagger}$ F. Margaroli, ${ }^{46 a, \dagger}$ P. Marino, ${ }^{41 \mathrm{a}, 4 \mathrm{ld}, \dagger}$ J. Martínez-Ortega, ${ }^{82, \$}$ K. Matera, ${ }^{22, \dagger}$ M. E. Mattson, ${ }^{52, \dagger}$ A. Mazzacane, ${ }^{15, \dagger}$ P. Mazzanti, ${ }^{6 a}{ }^{\dagger}$ R. McCarthy, ${ }^{12, \ddagger}$ C. L. McGivern, ${ }^{93, \dagger}$ R. McNulty, ${ }^{27, i, \dagger}$ A. Mehta, ${ }^{27, \dagger}$ P. Mehtala, ${ }^{21, \dagger}$

M. M. Meijer, ${ }^{83,84, \$}$ A. Melnitchouk, ${ }^{15,}$ D. Menezes, ${ }^{98, \$}$ P. G. Mercadante, ${ }^{57, \$}$ M. Merkin, ${ }^{85, \$}$ C. Mesropian, ${ }^{45, \dagger}$

A. Meyer, ${ }^{72, \dagger}$ J. Meyer, ${ }^{74, s s, \$}$ T. Miao, ${ }^{15, \dagger}$ F. Miconi,${ }^{70,}$ D. Mietlicki, ${ }^{31, \dagger}$ A. Mitra, ${ }^{1, \dagger}$ H. Miyake, ${ }^{49, \dagger}$ S. Moed,${ }^{15, \dagger}$ N. Moggi, ${ }^{6 a, \dagger}$ N. K. Mondal, ${ }^{79, *}$ C. S. Moon, ${ }^{25, \dagger}$ R. Moore, ${ }^{15, f f, g g, \dagger}$ M. J. Morello, ${ }^{41 \mathrm{a}, 41 \mathrm{~d}, \dagger}$ A. Mukherjee, ${ }^{15, \dagger}$ M. Mulhearn, ${ }^{12,+}$ Th. Muller, ${ }^{24, \dagger}$ P. Murat, ${ }^{15, \dagger}$ M. Mussini, ${ }^{6 a, 6 b, \dagger}$ J. Nachtman, ${ }^{15, m, \dagger}$ Y. Nagai, ${ }^{49, \dagger}$ J. Naganoma, ${ }^{51, \dagger}$ E. Nagy, ${ }^{66,}$ I. Nakano, ${ }^{36, \dagger}$ A. Napier, ${ }^{50, \dagger}$ M. Narain, ${ }^{118, \$}$ R. Nayyar, ${ }^{94, \$}$ H. A. Neal, ${ }^{31, \$}$ J. P. Negret, ${ }^{59, \sharp}$ J. Nett, ${ }^{47,}{ }^{\dagger}$ P. Neustroev, ${ }^{87, \$}$ H. T. Nguyen, ${ }^{122, \$}$ T. Nigmanov, ${ }^{42, \dagger}$ L. Nodulman ${ }^{2, \dagger}$ S. Y. Noh, ${ }^{25, \dagger}$ O. Norniella, ${ }^{22, \dagger}$ T. Nunnemann, ${ }^{76,}$ L. Oakes,${ }^{38, \dagger}$ S. H. Oh, ${ }^{14, \dagger}$ Y. D. Oh, ${ }^{25, \dagger}$ T. Okusawa, ${ }^{37, \dagger}$ R. Orava, ${ }^{21, \dagger}$ J. Orduna, ${ }^{118,}$ L. Ortolan, ${ }^{4, \dagger}$ N. Osman, ${ }^{66,}$ C. Pagliarone, ${ }^{48 a, \dagger}$ A. Pal, ${ }^{119, \dagger}$ E. Palencia, ${ }^{9, e, \dagger}$ P. Palni, ${ }^{34, \dagger}$ V. Papadimitriou, ${ }^{15 \dagger}$ N. Parashar, ${ }^{101,}$ V. Parihar, ${ }^{118,}$ S. K. Park, ${ }^{81,}$ W. Parker, ${ }^{53, \dagger}$ R. Partridge, ${ }^{118,00, \$}$ N. Parua, ${ }^{100, \$}$ A. Patwa, ${ }^{113, t t, \$}$ G. Pauletta, ${ }^{48 a, 48 b, 48 c, \dagger}$ M. Paulini, ${ }^{10, \dagger}$ C. Paus,${ }^{30, \dagger}$ B. Penning, ${ }^{92, \$}$ M. Perfilov, ${ }^{85, \$}$ Y. Peters, ${ }^{93, \$}$ K. Petridis, ${ }^{93, \neq}$ G. Petrillo, ${ }^{44, \$}$ P. Pétroff, ${ }^{67,}$ T. J. Phillips, ${ }^{14, \dagger}$ G. Piacentino, ${ }^{15, q, \dagger}$ E. Pianori, ${ }^{40, \dagger}$ J. Pilot, ${ }^{7, \dagger}$ K. Pitts, ${ }^{22, \dagger}$ C. Plager, ${ }^{8, \dagger}$ M.-A. Pleier, ${ }^{113, \ddagger}$ V. M. Podstavkov, ${ }^{15, \ddagger}$ L. Pondrom, ${ }^{53, \dagger}$ A. V. Popov, ${ }^{86,}$ S. Poprocki, ${ }^{15, f, \dagger}$ K. Potamianos, ${ }^{26, \dagger}$ A. Pranko, ${ }^{26, \dagger}$ M. Prewitt, ${ }^{121, \ddagger}$ D. Price, ${ }^{93, \star}$ N. Prokopenko, ${ }^{86, \$}$ F. Prokoshin, ${ }^{13, \text { aa }, \dagger}$ F. Ptohos, ${ }^{17, g, \dagger}$ G. Punzi, ${ }^{41 \mathrm{a}, 41 \mathrm{~b}, \dagger}$ J. Qian, ${ }^{31, \$}$ A. Quadt, ${ }^{74, \$}$ B. Quinn, ${ }^{107, \$}$ P. N. Ratoff, ${ }^{91,}$ I. Razumov, ${ }^{86, \$}$ I. Redondo Fernández, ${ }^{29, \dagger}$ P. Renton, ${ }^{38, \dagger}$ M. Rescigno, ${ }^{46 a, \dagger}$ F. Rimondi, ${ }^{6 a, *}, \dagger$ I. Ripp-Baudot, ${ }^{70,}$ L. Ristori, ${ }^{41 \mathrm{a}, 15, \dagger}$ F. Rizatdinova, ${ }^{116, \ddagger}$ A. Robson, ${ }^{19, \dagger}$ T. Rodriguez, ${ }^{40, \dagger}$ S. Rolli, ${ }^{50, \mathrm{~h}, \dagger} \mathrm{M}$. Rominsky, ${ }^{15, \ddagger}$ M. Ronzani, ${ }^{41 \mathrm{a}, 41 \mathrm{~b}, \dagger}$ R. Roser, ${ }^{15, \dagger}$ J. L. Rosner, ${ }^{11, \dagger}$ A. Ross, ${ }^{91, \dagger}$ C. Royon, ${ }^{62, \$}$ P. Rubinov, ${ }^{15, \dagger}$ R. Ruchti, ${ }^{102, \$}$ F. Ruffini, ${ }^{41 a, 41 c, \dagger}$ A. Ruiz, ${ }^{9, \dagger}$ J. Russ, ${ }^{10, \dagger}$ V. Rusu, ${ }^{15, \dagger}$ G. Sajot, ${ }^{65, \dagger}$ W. K. Sakumoto, ${ }^{44, \dagger}$ Y. Sakurai, ${ }^{51, \dagger}$ A. Sánchez-Hernández, ${ }^{82, \ddagger}$ M. P. Sanders, ${ }^{76, \sharp}$ L. Santi, ${ }^{48 a, 48 b, 48 c, \dagger}$ A. S. Santos, ${ }^{55, r r, \sharp}$ K. Sato, ${ }^{49, \dagger}$ G. Savage, ${ }^{15, \sharp}$ V. Saveliev, ${ }^{15, v, \dagger}$ M. Savitskyi, ${ }^{90, \sharp}$ A. Savoy-Navarro, ${ }^{15, z, \dagger}$ L. Sawyer,${ }^{105, \$}$ T. Scanlon, ${ }^{92, *}$ R. D. Schamberger, ${ }^{112, \ddagger}$ Y. Scheglov, ${ }^{87, *}, *$ H. Schellman, ${ }^{117,99, *}$

P. Schlabach, ${ }^{15, \dagger}$ E. E. Schmidt, ${ }^{15, \dagger}$ M. Schott, ${ }^{75}$ C. Schwanenberger, ${ }^{93, \dagger}$ T. Schwarz, ${ }^{31, \dagger}$ R. Schwienhorst, ${ }^{32, \$}$

L. Scodellaro, ${ }^{9, \dagger}$ F. Scuri, ${ }^{41 a, \dagger}$ S. Seidel, ${ }^{34, \dagger}$ Y. Seiya, ${ }^{37, \dagger}$ J. Sekaric, ${ }^{104, *}$ A. Semenov, ${ }^{13, \dagger}$ H. Severini, ${ }^{115, \$}$ F. Sforza, ${ }^{41 a, 41 b, \dagger}$ E. Shabalina, ${ }^{74, \$}$ S. Z. Shalhout, ${ }^{7, \dagger}$ V. Shary, ${ }^{69,}$ S. Shaw, ${ }^{93, \ddagger}$ A. A. Shchukin, ${ }^{86,}$ T. Shears, ${ }^{27, \dagger}$ P. F. Shepard, ${ }^{42, \dagger}$ M. Shimojima, ${ }^{49, \mathrm{u}, \dagger}$ O. Shkola, ${ }^{90, \$}$ M. Shochet, ${ }^{11, \dagger}$ I. Shreyber-Tecker, ${ }^{33, \dagger}$ V. Simak, ${ }^{61, \$}$ A. Simonenko, ${ }^{13, \dagger}$ P. Skubic, ${ }^{115, \$}$ P. Slattery, ${ }^{44}$ K. Sliwa, ${ }^{50 \dagger}$ J. R. Smith, ${ }^{7, \dagger}$ F. D. Snider, ${ }^{15, \dagger}$ G. R. Snow, ${ }^{108, \ddagger}$ J. Snow, ${ }^{114, \$}$ S. Snyder, ${ }^{113, \$}$

S. Söldner-Rembold, ${ }^{93,}$ H. Song, ${ }^{42, \dagger}$ L. Sonnenschein, ${ }^{72, \$}$ V. Sorin, ${ }^{4, \dagger}$ K. Soustruznik, ${ }^{60,}$ R. St. Denis, ${ }^{19, *, \dagger}$ M. Stancari, ${ }^{15, \dagger}$ J. Stark, ${ }^{65, \$}$ N. Stefaniuk, ${ }^{90, \ddagger}$ D. Stentz, ${ }^{15, w, \dagger}$ D. A. Stoyanova, ${ }^{86,}$ M. Strauss, ${ }^{115, \ddagger}$ J. Strologas, ${ }^{34, \dagger}$ Y. Sudo, ${ }^{49, \dagger}$

A. Sukhanov, ${ }^{15 \dagger}$ I. Suslov, ${ }^{13, \dagger}$ L. Suter, ${ }^{93,}$ P. Svoisky, ${ }^{122, \dagger}$ K. Takemasa, ${ }^{49, \dagger}$ Y. Takeuchi, ${ }^{49, \dagger}$ J. Tang, ${ }^{11, \dagger}$ M. Tecchio, ${ }^{31, \dagger}$ P. K. Teng, ${ }^{1,}$ J. Thom, ${ }^{15, f, \dagger}$ E. Thomson, ${ }^{40, \dagger}$ V. Thukral, ${ }^{47, \dagger}$ M. Titov, ${ }^{69, \$}$ D. Toback, ${ }^{47, \dagger}$ S. Tokar, ${ }^{12, \dagger}$ V. V. Tokmenin, ${ }^{13, \$}$ K. Tollefson, ${ }^{32, \dagger}$ T. Tomura,${ }^{49, \dagger}$ D. Tonelli, ${ }^{15, e, \dagger}$ S. Torre,${ }^{17, \dagger}$ D. Torretta, ${ }^{15, \dagger}$ P. Totaro, ${ }^{39 a, \dagger}$ M. Trovato, ${ }^{41 \mathrm{a}, 41 \mathrm{~d}, \dagger}$ Y.-T. Tsai, ${ }^{44, \$}$ D. Tsybychev, ${ }^{112, \$}$ B. Tuchming, ${ }^{69, \$}$ C. Tully, ${ }^{110, \ddagger}$ F. Ukegawa, ${ }^{49, \dagger}$ S. Uozumi, ${ }^{25, \dagger}$ L. Uvarov, ${ }^{87, \ddagger}$ S. Uvarov, ${ }^{87}$,

${ }^{\ddagger}$ S. Uzunyan, ${ }^{98,}$ R. Van Kooten, ${ }^{100, \$}$ W. M. van Leeuwen, ${ }^{83, \$}$ N. Varelas, ${ }^{97, \$}$ E. W. Varnes, ${ }^{94, \ddagger}$ I. A. Vasilyev, ${ }^{86, \$}$

F. Vázquez, ${ }^{16,1, \dagger}$ G. Velev, ${ }^{15, \dagger}$ C. Vellidis, ${ }^{15, \dagger}$ A. Y. Verkheev, ${ }^{13, \dagger}$ C. Vernieri, ${ }^{41 a, 41 d, \dagger}$ L. S. Vertogradov, ${ }^{13, \$}$ M. Verzocchi, ${ }^{15, \$}$ M. Vesterinen, ${ }^{93, \$}$ M. Vidal, ${ }^{43, \dagger}$ D. Vilanova, ${ }^{69, \$}$ R. Vilar, ${ }^{9, \dagger}$ J. Vizán, ${ }^{9, d d, \dagger}$ M. Vogel, ${ }^{34, \dagger}$ P. Vokac, ${ }^{61, \ddagger}$ G. Volpi, ${ }^{17, \dagger}$ P. Wagner, ${ }^{40, \dagger}$ H. D. Wahl, ${ }^{96, \$}$ R. Wallny, ${ }^{15, j, \dagger}$ C. Wang, ${ }^{58, \$}$ M. H. L. S. Wang, ${ }^{15, \$}$ S. M. Wang, ${ }^{1, \dagger}$ J. Warchol, ${ }^{102, *, *}$

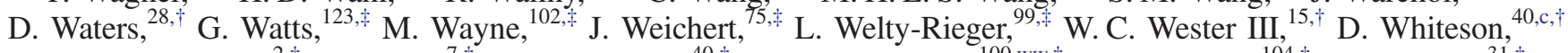
A. B. Wicklund, ${ }^{2, \dagger}$ S. Wilbur, ${ }^{7, \dagger}$ H. H. Williams, ${ }^{40, \dagger}$ M. R. J. Williams, ${ }^{100, w w, \$}$ G. W. Wilson, ${ }^{104, \$}$ J. S. Wilson, ${ }^{31, \dagger}$ P. Wilson, ${ }^{15, \dagger}$ B. L. Winer, ${ }^{35, \dagger}$ P. Wittich, ${ }^{15, f \dagger}$ M. Wobisch, ${ }^{105, \$}$ S. Wolbers, ${ }^{15, \dagger}$ H. Wolfmeister, ${ }^{35,}$ D. R. Wood, ${ }^{106, \$}$ T. Wright ${ }^{31, \dagger}$ X. Wu, ${ }^{18, \dagger}$ Z. Wu,${ }^{5, \dagger}$ T. R. Wyatt, ${ }^{93, \dagger}$ Y. Xiang, ${ }^{58, \$}$ Y. Xie ${ }^{15, \$}$ R. Yamada ${ }^{15, \$}$ K. Yamamoto, ${ }^{37, \dagger}$ D. Yamato, ${ }^{37, \dagger}$ S. Yang, ${ }^{58,+}$ T. Yang, ${ }^{15}{ }^{\dagger}$ U. K. Yang, ${ }^{25, \dagger}$ Y. C. Yang, ${ }^{25, \dagger}$ W.-M. Yao, ${ }^{26,}{ }^{\dagger}$ T. Yasuda, ${ }^{15,}$ Y. A. Yatsunenko, ${ }^{13, \ddagger}$ W. Ye, ${ }^{112, \$}$ Z. Ye, ${ }^{15,}$ G. P. Yeh, ${ }^{15 \dagger}$ K. Yi, ${ }^{15, m, \dagger}$ H. Yin, ${ }^{15,}$ K. Yip, ${ }^{113, \$}$ J. Yoh, ${ }^{15, \dagger}$ K. Yorita, ${ }^{51,}{ }^{\dagger}$ T. Yoshida, ${ }^{37, k, \dagger}$ S. W. Youn, ${ }^{15, \$}$ G. B. Yu, ${ }^{14, \dagger}$ I. Yu, ${ }^{25, \dagger}$ J. M. Yu, ${ }^{31,}$ A. M. Zanetti, ${ }^{48 a, \dagger}$ Y. Zeng, ${ }^{14, \dagger}{ }^{\dagger}$. Zennamo, ${ }^{11, \$}$ T. G. Zhao, ${ }^{93,}$ B. Zhou, ${ }^{31, \$}$ C. Zhou, ${ }^{14, \dagger}$ J. Zhu, ${ }^{31,}$ M. Zielinski, ${ }^{44, \$}$ D. Zieminska, ${ }^{100, \$}$ L. Zivkovic, ${ }^{68, y y, \$}$ and S. Zucchelli ${ }^{6 a, 6 b, \dagger}$ 
$(\mathrm{CDF} \text { Collaboration })^{\dagger}$

(D0 Collaboration)

${ }^{1}$ Institute of Physics, Academia Sinica, Taipei, Taiwan 11529, Republic of China

${ }^{2}$ Argonne National Laboratory, Argonne, Illinois 60439, USA

${ }^{3}$ University of Athens, 15771 Athens, Greece

${ }^{4}$ Institut de Fisica d'Altes Energies, ICREA, Universitat Autonoma de Barcelona, E-08193 Bellaterra (Barcelona), Spain

${ }^{5}$ Baylor University, Waco, Texas 76798, USA

${ }^{6 a}$ Istituto Nazionale di Fisica Nucleare Bologna, I-40127 Bologna, Italy

${ }^{6 \mathrm{~b}}$ University of Bologna, I-40127 Bologna, Italy

${ }^{7}$ University of California, Davis, Davis, California 95616, USA

${ }^{8}$ University of California, Los Angeles, Los Angeles, California 90024, USA

${ }^{9}$ Instituto de Fisica de Cantabria, CSIC-University of Cantabria, 39005 Santander, Spain

${ }^{10}$ Carnegie Mellon University, Pittsburgh, Pennsylvania 15213, USA

${ }^{11}$ Enrico Fermi Institute, University of Chicago, Chicago, Illinois 60637, USA

${ }^{12}$ Comenius University, 84248 Bratislava, Slovakia; Institute of Experimental Physics, 04001 Kosice, Slovakia

${ }^{13}$ Joint Institute for Nuclear Research, RU-141980 Dubna, Russia

${ }^{14}$ Duke University, Durham, North Carolina 27708, USA

${ }^{15}$ Fermi National Accelerator Laboratory, Batavia, Illinois 60510, USA

${ }^{16}$ University of Florida, Gainesville, Florida 32611, USA

${ }^{17}$ Laboratori Nazionali di Frascati, Istituto Nazionale di Fisica Nucleare, I-00044 Frascati, Italy

${ }^{18}$ University of Geneva, CH-1211 Geneva 4, Switzerland

${ }^{19}$ Glasgow University, Glasgow G12 8QQ, United Kingdom

${ }^{20}$ Harvard University, Cambridge, Massachusetts 02138, USA

${ }^{21}$ Division of High Energy Physics, Department of Physics, University of Helsinki, FIN-00014 Helsinki,

Finland; Helsinki Institute of Physics, FIN-00014 Helsinki, Finland

${ }^{22}$ University of Illinois, Urbana, Illinois 61801, USA

${ }^{23}$ The Johns Hopkins University, Baltimore, Maryland 21218, USA

${ }^{24}$ Institut für Experimentelle Kernphysik, Karlsruhe Institute of Technology, D-76131 Karlsruhe, Germany

${ }^{25}$ Center for High Energy Physics: Kyungpook National University, Daegu 702-701, Korea;

Seoul National University, Seoul 151-742, Korea; Sungkyunkwan University, Suwon 440-746, Korea;

Korea Institute of Science and Technology Information, Daejeon 305-806, Korea;

Chonnam National University, Gwangju 500-757, Korea; Chonbuk National University,

Jeonju 561-756, Korea; Ewha Womans University, Seoul, 120-750, Korea

${ }^{26}$ Ernest Orlando Lawrence Berkeley National Laboratory, Berkeley, California 94720, USA

${ }^{27}$ University of Liverpool, Liverpool L69 7ZE, United Kingdom

${ }^{28}$ University College London, London WC1E 6BT, United Kingdom

${ }^{29}$ Centro de Investigaciones Energeticas Medioambientales y Tecnologicas, E-28040 Madrid, Spain

${ }^{30}$ Massachusetts Institute of Technology, Cambridge, Massachusetts 02139, USA

${ }^{31}$ University of Michigan, Ann Arbor, Michigan 48109, USA

${ }^{32}$ Michigan State University, East Lansing, Michigan 48824, USA

${ }^{33}$ Institution for Theoretical and Experimental Physics, ITEP, Moscow 117259, Russia

${ }^{34}$ University of New Mexico, Albuquerque, New Mexico 87131, USA

${ }^{35}$ The Ohio State University, Columbus, Ohio 43210, USA

${ }^{36}$ Okayama University, Okayama 700-8530, Japan

${ }^{37}$ Osaka City University, Osaka 558-8585, Japan

${ }^{38}$ University of Oxford, Oxford OX1 3RH, United Kingdom

${ }^{39 a}$ Istituto Nazionale di Fisica Nucleare, Sezione di Padova, I-35131 Padova, Italy

${ }^{39 b}$ University of Padova, I-35131 Padova, Italy

${ }^{40}$ University of Pennsylvania, Philadelphia, Pennsylvania 19104, USA

${ }^{41 \mathrm{a}}$ Istituto Nazionale di Fisica Nucleare Pisa, I-56127 Pisa, Italy

${ }^{41 \mathrm{~b}}$ University of Pisa, I-56127 Pisa, Italy

${ }^{41 c}$ University of Siena, I-56127 Pisa, Italy

${ }^{41 \mathrm{~d}}$ Scuola Normale Superiore, I-56127 Pisa, Italy

${ }^{41 \mathrm{e}}$ INFN Pavia, I-27100 Pavia, Italy

${ }^{41 \mathrm{f}}$ University of Pavia, I-27100 Pavia, Italy 
${ }^{42}$ University of Pittsburgh, Pittsburgh, Pennsylvania 15260, USA

${ }^{43}$ Purdue University, West Lafayette, Indiana 47907, USA

${ }^{44}$ University of Rochester, Rochester, New York 14627, USA

${ }^{45}$ The Rockefeller University, New York, New York 10065, USA

${ }^{46 a}$ Istituto Nazionale di Fisica Nucleare, Sezione di Roma 1, I-00185 Roma, Italy

${ }^{46 \mathrm{~b}}$ Sapienza Università di Roma, I-00185 Roma, Italy

${ }^{47}$ Mitchell Institute for Fundamental Physics and Astronomy, Texas A\&M University, College Station, Texas 77843, USA

${ }^{48 \mathrm{a}}$ Istituto Nazionale di Fisica Nucleare Trieste, I-34127 Trieste, Italy

${ }^{48 \mathrm{~b}}$ Gruppo Collegato di Udine, I-33100 Udine, Italy

${ }^{48 \mathrm{c}}$ University of Udine, I-33100 Udine, Italy

${ }^{48 \mathrm{~d}}$ University of Trieste, I-34127 Trieste, Italy

${ }^{49}$ University of Tsukuba, Tsukuba, Ibaraki 305, Japan

${ }^{50}$ Tufts University, Medford, Massachusetts 02155, USA

${ }^{51}$ Waseda University, Tokyo 169, Japan

${ }^{52}$ Wayne State University, Detroit, Michigan 48201, USA

${ }^{53}$ University of Wisconsin-Madison, Madison, Wisconsin 53706, USA

${ }^{54}$ Yale University, New Haven, Connecticut 06520, USA

${ }^{55}$ LAFEX, Centro Brasileiro de Pesquisas Físicas, Rio de Janeiro, RJ 22290, Brazil

${ }^{56}$ Universidade do Estado do Rio de Janeiro, Rio de Janeiro, RJ 20550, Brazil

${ }^{57}$ Universidade Federal do ABC, Santo André, SP 09210, Brazil

${ }^{58}$ University of Science and Technology of China, Hefei 230026, People's Republic of China

${ }^{59}$ Universidad de los Andes, Bogotá, 111711, Colombia

${ }^{60}$ Charles University, Faculty of Mathematics and Physics, Center for Particle Physics, 11636 Prague 1, Czech Republic

${ }^{61}$ Czech Technical University in Prague, 11636 Prague 6, Czech Republic

${ }^{62}$ Institute of Physics, Academy of Sciences of the Czech Republic, 18221 Prague, Czech Republic

${ }^{63}$ Universidad San Francisco de Quito, Quito 170157, Ecuador

${ }^{64}$ LPC, Université Blaise Pascal, CNRS/IN2P3, Clermont, F-63178 Aubière Cedex, France

${ }^{65}$ LPSC, Université Joseph Fourier Grenoble 1, CNRS/IN2P3, Institut National Polytechnique de Grenoble, F-38026 Grenoble Cedex, France

${ }^{66}$ CPPM, Aix-Marseille Université, CNRS/IN2P3, F-13288 Marseille Cedex 09, France

${ }^{67}$ LAL, Univ. Paris-Sud, CNRS/IN2P3, Université Paris-Saclay, F-91898 Orsay Cedex, France

${ }^{68}$ LPNHE, Universités Paris VI and VII, CNRS/IN2P3, F-75005 Paris, France

${ }^{69}$ CEA Saclay, Irfu, SPP, F-91191 Gif-Sur-Yvette Cedex, France

${ }^{70} I P H C$, Université de Strasbourg, CNRS/IN2P3, F-67037 Strasbourg, France

${ }^{71} I P N L$, Université Lyon 1, CNRS/IN2P3, F-69622 Villeurbanne Cedex, France and Université de Lyon, F-69361 Lyon CEDEX 07, France

${ }^{72}$ III. Physikalisches Institut A, RWTH Aachen University, 52056 Aachen, Germany

${ }^{73}$ Physikalisches Institut, Universität Freiburg, 79085 Freiburg, Germany

${ }^{74}$ II. Physikalisches Institut, Georg-August-Universität Göttingen, 37073 Göttingen, Germany

${ }^{75}$ Institut für Physik, Universität Mainz, 55099 Mainz, Germany

${ }^{76}$ Ludwig-Maximilians-Universität München, 80539 München, Germany

${ }^{77}$ Panjab University, Chandigarh 160014, India

${ }^{78}$ Delhi University, Delhi-110 007, India

${ }^{79}$ Tata Institute of Fundamental Research, Mumbai-400 005, India

${ }^{80}$ University College Dublin, Dublin 4, Ireland

${ }^{81}$ Korea Detector Laboratory, Korea University, Seoul, 02841, Korea

${ }^{82}$ CINVESTAV, Mexico City 07360, Mexico

${ }^{83}$ Nikhef, Science Park, 1098 XG Amsterdam, the Netherlands

${ }^{84}$ Radboud University Nijmegen, 6525 AJ Nijmegen, the Netherlands

${ }^{85}$ Moscow State University, Moscow 119991, Russia

${ }^{86}$ Institute for High Energy Physics, Protvino, Moscow region 142281, Russia

${ }^{87}$ Petersburg Nuclear Physics Institute, St. Petersburg 188300, Russia

${ }^{88}$ Institució Catalana de Recerca i Estudis Avançats (ICREA) and Institut de Física d'Altes Energies

(IFAE), 08193 Bellaterra (Barcelona), Spain

${ }^{89}$ Uppsala University, 75105 Uppsala, Sweden

${ }^{90}$ Taras Shevchenko National University of Kyiv, Kiev, 01601, Ukraine

${ }^{91}$ Lancaster University, Lancaster LA1 4YB, United Kingdom

${ }^{92}$ Imperial College London, London SW7 2AZ, United Kingdom 


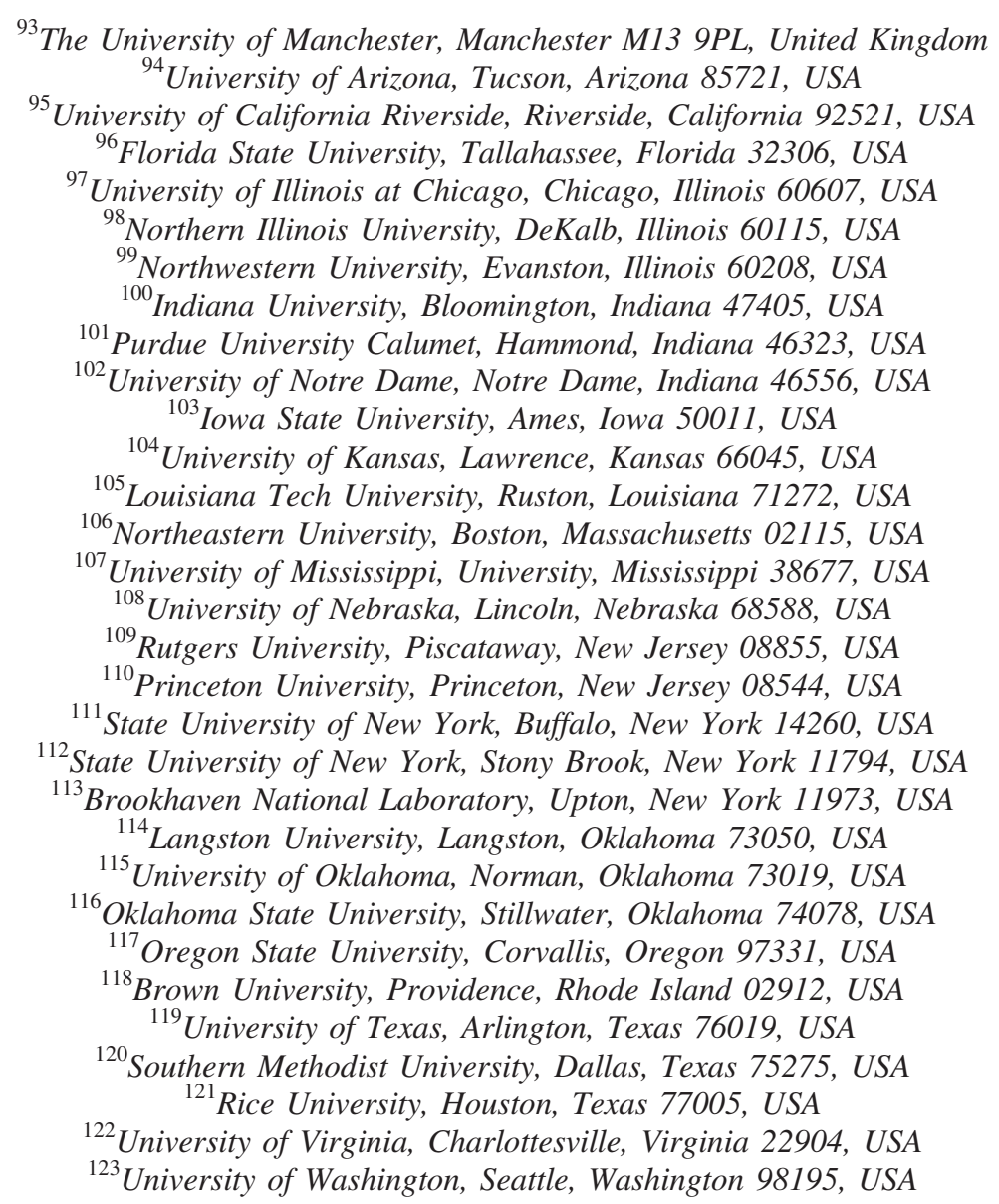

\footnotetext{
${ }^{*}$ Deceased.

${ }^{a}$ Also at University of British Columbia, Vancouver, BC V6T 1Z1, Canada.

${ }^{\mathrm{b}}$ Also at Istituto Nazionale di Fisica Nucleare, Sezione di Cagliari, 09042 Monserrato (Cagliari), Italy.

${ }^{c}$ Also at University of California Irvine, Irvine, CA 92697, USA.

dAlso at Institute of Physics, Academy of Sciences of the Czech Republic, 182 21, Czech Republic.

eAlso at CERN, CH-1211 Geneva, Switzerland.

${ }^{\mathrm{f}}$ Also at Cornell University, Ithaca, NY 14853, USA.

${ }^{\mathrm{g}}$ Also at University of Cyprus, Nicosia CY-1678, Cyprus.

${ }^{\mathrm{h}}$ Also at Office of Science, U.S. Department of Energy, Washington, DC 20585, USA.

${ }^{\mathrm{i}}$ Also at University College Dublin, Dublin 4, Ireland.

${ }^{\mathrm{j}}$ Also at ETH, 8092 Zürich, Switzerland.

${ }^{\mathrm{k}}$ Also at University of Fukui, Fukui City, Fukui Prefecture, Japan 910-0017.

${ }^{1}$ Also at Universidad Iberoamericana, Lomas de Santa Fe, México, C.P. 01219, Distrito Federal.

${ }^{\mathrm{m}}$ Also at University of Iowa, Iowa City, IA 52242, USA.

${ }^{\mathrm{n}}$ Also at Kinki University, Higashi-Osaka City, Japan 577-8502.

${ }^{\circ}$ Also at Kansas State University, Manhattan, KS 66506, USA.

${ }^{\mathrm{p}}$ Also at Brookhaven National Laboratory, Upton, NY 11973, USA.

${ }^{\mathrm{q}}$ Also at Istituto Nazionale di Fisica Nucleare, Sezione di Lecce, Via Arnesano, I-73100 Lecce, Italy.

${ }^{\mathrm{r}}$ Also at Queen Mary, University of London, London, E1 4NS, United Kingdom.

${ }^{\mathrm{s}}$ Also at University of Melbourne, Victoria 3010, Australia.

${ }^{t}$ Also at Muons, Inc., Batavia, IL 60510, USA.

uAlso at Nagasaki Institute of Applied Science, Nagasaki 851-0193, Japan.

${ }^{v}$ Also at National Research Nuclear University, Moscow 115409, Russia.

${ }^{\mathrm{w}}$ Also at Northwestern University, Evanston, IL 60208, USA.

${ }^{\mathrm{x}}$ Also at University of Notre Dame, Notre Dame, IN 46556, USA.

${ }^{\mathrm{y}}$ Also at Universidad de Oviedo, E-33007 Oviedo, Spain.

${ }^{\mathrm{z}}$ Also at CNRS-IN2P3, Paris, F-75205 France.

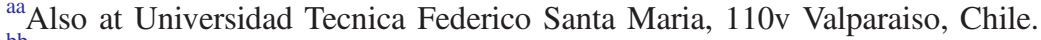

${ }^{\mathrm{bb}}$ Also at Sejong University, Seoul 143-747, Korea.

${ }^{\mathrm{cc}}$ Also at The University of Jordan, Amman 11942, Jordan.
} 
(Received 19 January 2018; published 28 June 2018)

Drell-Yan lepton pairs produced in the process $p \bar{p} \rightarrow \ell^{+} \ell^{-}+X$ through an intermediate $\gamma^{*} / Z$ boson have an asymmetry in their angular distribution related to the spontaneous symmetry breaking of the electroweak force and the associated mixing of its neutral gauge bosons. The CDF and D0 experiments have measured the effective-leptonic electroweak mixing parameter $\sin ^{2} \theta_{\text {eff }}^{\text {lept }}$ using electron and muon pairs selected from the full Tevatron proton-antiproton data sets collected in 2001-2011, corresponding to $9-10 \mathrm{fb}^{-1}$ of integrated luminosity. The combination of these measurements yields the most precise result from hadron colliders, $\sin ^{2} \theta_{\mathrm{eff}}^{\text {lept }}=0.23148 \pm 0.00033$. This result is consistent with, and approaches in precision, the best measurements from electron-positron colliders. The standard model inference of the on-shell electroweak mixing parameter $\sin ^{2} \theta_{W}$, or equivalently the $W$-boson mass $M_{W}$, using the ZFITTER software package yields $\sin ^{2} \theta_{W}=0.22324 \pm 0.00033$ or equivalently, $M_{W}=80.367 \pm 0.017 \mathrm{GeV} / c^{2}$.

DOI: 10.1103/PhysRevD.97.112007

\section{INTRODUCTION}

At the Fermilab Tevatron proton-antiproton $(p \bar{p})$ collider, Drell-Yan [1] lepton $(\ell)$ pairs are produced in the process $p \bar{p} \rightarrow \ell^{+} \ell^{-}+X$ through an intermediate $\gamma^{*} / Z$ boson, where $X$ represents inclusively any other collision products. The forward-backward asymmetry in the polarangle distribution of the $\ell^{-}$in the Collins-Soper (CS) frame [2] as a function of the $\ell^{+} \ell^{-}$-pair invariant mass is directly sensitive to the effective-leptonic electroweak mixing parameter $\sin ^{2} \theta_{\text {eff }}^{\text {lept }}$. The effective-leptonic parameter is measured using electron and muon pairs $(\ell=e$ and $\mu)$. The electroweak-mixing parameter $\sin ^{2} \theta_{W}$ [3] is obtained indirectly in the context of standard model (SM) calculations with the following input parameters: the fine structure constant, the Fermi constant, the strong interaction coupling constant, and the masses of the top quark, $Z$ boson, and Higgs boson. In this SM context, $\sin ^{2} \theta_{W}$ and the $W$-boson mass are related, and a comparison of the $W$ boson mass inferred from $\sin ^{2} \theta_{W}$ to the directly measured mass tests the consistency of the SM. Such tests require precision measurements of $\sin ^{2} \theta_{\mathrm{eff}}^{\text {lept }}$, and results from hadron colliders such as the Tevatron are complementary to those from electron-positron colliders.

The Drell-Yan process and the production of quark pairs in high-energy $e^{+} e^{-}$collisions are analogous processes: $q \bar{q} \rightarrow \ell^{+} \ell^{-}$and $e^{+} e^{-} \rightarrow q \bar{q}$. The $\sin ^{2} \theta_{\text {eff }}^{\text {lept }}$ parameter of

${ }^{\mathrm{dd}}$ Also at Universite catholique de Louvain, 1348 Louvain-La-Neuve, Belgium.

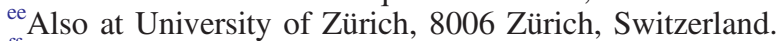

${ }^{\mathrm{ff}}$ Also at Massachusetts General Hospital, Boston, MA 02114 USA.

${ }^{\mathrm{gg}}$ Also at Harvard Medical School, Boston, MA 02114 USA.

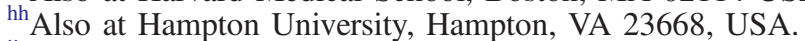

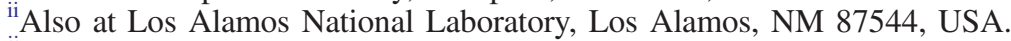

${ }^{j j}$ Also at Università degli Studi di Napoli Federico II, I-80138 Napoli, Italy

${ }^{\mathrm{kk}}$ Also at Augustana University, Sioux Falls, SD 57197, USA.

${ }^{11}$ Also at The University of Liverpool, Liverpool L69 3BX, UK.

${ }^{m m}$ Also at Deutsches Elektronen-Synchrotron (DESY), Notkestrase 85, Germany.

${ }^{n n}$ Also at Consejo Nacional de Ciencia y Tecnologia (Conacyt), M-03940 Mexico City, Mexico.

${ }^{\circ 0}$ Also at SLAC, Menlo Park, CA 94025, USA.

${ }^{\mathrm{pp}}$ Also at University College London, London WC1E 6BT, UK.

${ }^{\mathrm{qq}} \mathrm{Also}$ at Centro de Investigacion en Computacion - IPN, CP 07738 Mexico City, Mexico.

${ }^{\mathrm{rr}}$ Also at Universidade Estadual Paulista, S ao Paulo, SP 01140, Brazil.

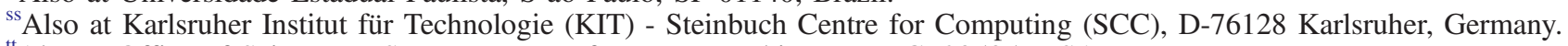

"Also at Office of Science, U.S. Department of Energy, Washington, D.C. 20585, USA.

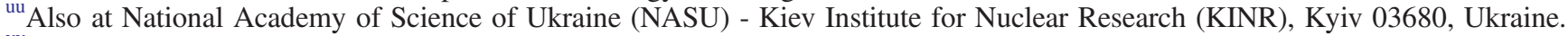

${ }^{\mathrm{vv}}$ Also at University of Maryland, College Park, MD 20742, USA.

${ }^{w w}$ Also at European Organization for Nuclear Research (CERN), CH-1211 Genéve 23, Switzerland.

${ }^{\mathrm{xx}}$ Also at Purdue University, West Lafayette, IN 47907, USA.

${ }^{\text {yy }}$ Also at Institute of Physics, Belgrade, CS-11080 Belgrade, Serbia.

${ }^{\mathrm{zz}}$ Also at P. N. Lebedev Physical Institute of the Russian Academy of Sciences, 119991, Moscow, Russia

Published by the American Physical Society under the terms of the Creative Commons Attribution 4.0 International license. Further distribution of this work must maintain attribution to the author(s) and the published article's title, journal citation, and DOI. Funded by $S C O A P^{3}$. 
processes involving leptons has been investigated at the LEP-1 and SLC $[4,5] e^{+} e^{-}$colliders operating on or in the vicinity of the $Z$-boson pole mass, and at the Tevatron [6-13] and LHC [14-16] hadron colliders. Investigations at hadron colliders use Drell-Yan pairs whose range of invariant masses about the $Z$-boson resonant peak is broad relative to the $e^{+} e^{-}$collider investigations. The mixing parameter has been accurately measured at the LEP-1 and SLC colliders, where processes with leptons in the final state are also used. The combined average of six measurements from these lepton colliders [4] yields a value of $0.23149 \pm 0.00016$ [5]. However, a 3.2 standard-deviation difference exists between the two most precise individual measurements. The combined measurement of the $b$-quark forward-backward asymmetry $\left(A_{\mathrm{FB}}^{0, \mathrm{~b}}\right)$ with the LEP-1 detectors yields $\sin ^{2} \theta_{\text {eff }}^{\text {lept }}=0.23221 \pm 0.00029$, while the SLD left-right polarization asymmetry of $Z$-boson production $\left(\mathcal{A}_{\ell}\right)$ yields $\sin ^{2} \theta_{\mathrm{eff}}^{\text {lept }}=0.23098 \pm 0.00026$. This provides a strong motivation for an accurate determination of $\sin ^{2} \theta_{\text {eff }}^{\text {lept }}$ by the Tevatron experiments.

\section{A. Electroweak couplings}

The production of Drell-Yan lepton pairs at the Born level proceeds through two parton-level processes,

$$
q \bar{q} \rightarrow \gamma^{*} \rightarrow \ell^{+} \ell^{-} \quad \text { and } \quad q \bar{q} \rightarrow Z \rightarrow \ell^{+} \ell^{-},
$$

where the $q$ and $\bar{q}$ are a quark and antiquark, respectively, that originate from the colliding hadrons. The virtual photon couples the vector currents of the incoming and outgoing fermions $(f)$, and the spacetime structure of the photon-fermion interaction vertex may be represented as $\left\langle\bar{f}\left|Q_{f} \gamma_{\mu}\right| f\right\rangle$, where $Q_{f}$, the strength of the coupling, is the fermion charge (in units of $e$ ), and $|f\rangle$ is the spinor for fermion $f$. The interaction vertex of a fermion with a $Z$ boson contains both vector $(V)$ and axial-vector $(A)$ current components, and its structure is $\left\langle\bar{f}\left|g_{V}^{f} \gamma_{\mu}+g_{A}^{f} \gamma_{\mu} \gamma_{5}\right| f\right\rangle$. The Born-level coupling strengths are

$$
g_{V}^{f}=T_{3}^{f}-2 Q_{f} \sin ^{2} \theta_{W} \quad \text { and } \quad g_{A}^{f}=T_{3}^{f},
$$

where $T_{3}^{f}$ is the third component of the fermion weak isospin, which is $T_{3}^{f}=\frac{1}{2}\left(-\frac{1}{2}\right)$ for positively (negatively) charged fermions. Radiative corrections alter the Born-level couplings into effective couplings. At the Born level in the $\mathrm{SM}$, and in all orders of the on-shell renormalization scheme [17], the $\sin ^{2} \theta_{W}$ parameter is related to the $W$ boson mass $M_{W}$ and the $Z$-boson mass $M_{Z}$ by $\sin ^{2} \theta_{W}=1-M_{W}^{2} / M_{Z}^{2}$. Since the $Z$-boson mass is accurately known (to $\pm 0.0021 \mathrm{GeV} / c^{2}[4,5]$ ), the inference of the on-shell $\sin ^{2} \theta_{W}$ is equivalent to an indirect $W$-boson mass measurement. The angular distributions of $\ell^{+} \ell^{-}$pairs in the final state of the Drell-Yan process and of $\ell^{+} \ell^{-}$ or $q \bar{q}$ pairs in the final state of $e^{+} e^{-}$collisions are sensitive to the effective $\sin ^{2} \theta_{W}$ parameter at the lepton vertex, $\sin ^{2} \theta_{\text {eff }}^{\text {lept }}$.

\section{B. The forward-backward asymmetry}

The rapidity, transverse momentum, and mass of a particle or a system of particles are represented by $y$, $p_{\mathrm{T}}$, and $M$, respectively. The energy and momentum of particles are represented as $E$ and $\vec{p}$, respectively. In the laboratory frame, the $p \bar{p}$ collision axis is the $z_{\text {lab }}$ axis, with the positive direction defined to be along the direction of the proton. The transverse component of any vector, such as the momentum vector, is defined relative to that axis. The rapidity is $y=\frac{1}{2} \ln \left[\left(E+p_{\mathrm{z}}\right) /\left(E-p_{\mathrm{z}}\right)\right]$, where $p_{\mathrm{z}}$ is the component of the momentum vector along the $z_{\text {lab }}$ axis.

The angular kinematic properties of leptons from the Drell-Yan process are defined in the rest frame of the exchanged $\gamma^{*} / Z$ boson. The $\ell^{-}$direction is chosen to define the polar and azimuthal angles of the lepton pair, which are denoted as $\vartheta$ and $\varphi$, respectively. The ideal positive $z$ axis coincides with the direction of the incoming quark so that the definition of $\vartheta$ parallels the definition used in $e^{+} e^{-}$collisions at LEP $[4,5]$. This frame is approximated by the CS rest frame [2] for $p \bar{p}$ collisions, depicted in Fig. 1.

The CS frame angle $\vartheta$ is reconstructed using the following laboratory-frame quantities: the lepton energies, the lepton momenta along the beam line, the dilepton invariant mass, $M$, and the dilepton transverse momentum, $p_{\mathrm{T}}$. The polar angle of the negatively charged lepton is calculated from

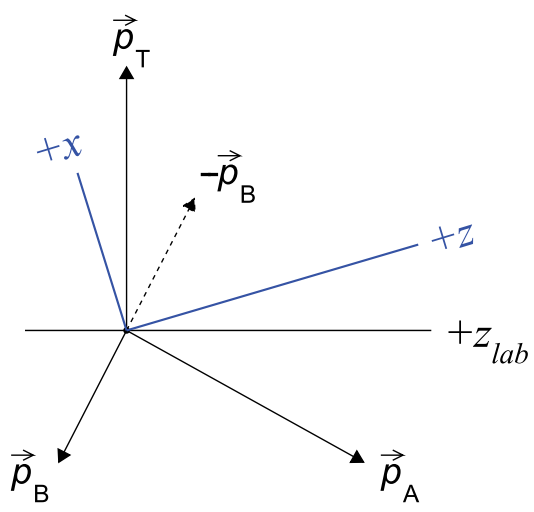

FIG. 1. Representation of the Collins-Soper coordinate axes $(x, z)$ in the lepton-pair rest frame relative to the laboratory $z$ axis $\left(z_{\text {lab }}\right)$. These three axes are in the plane formed by the proton $\left(\vec{p}_{\mathrm{A}}\right)$ and antiproton $\left(\vec{p}_{\mathrm{B}}\right)$ momentum vectors in the lepton-pair rest frame. The $z$ axis is the angular bisector of $\vec{p}_{\mathrm{A}}$ and $-\vec{p}_{\mathrm{B}}$. The $y$ axis is along the direction of $\vec{p}_{\mathrm{B}} \times \vec{p}_{\mathrm{A}}$, and the $x$ axis is in the direction opposite to the transverse component of $\vec{p}_{\mathrm{A}}+\vec{p}_{\mathrm{B}}$. 


$$
\cos \vartheta=\frac{l_{+}^{-} l_{-}^{+}-l_{-}^{-} l_{+}^{+}}{M \sqrt{M^{2}+p_{\mathrm{T}}^{2}}},
$$

where $l_{ \pm}=\left(E \pm p_{z}\right)$ and the $+(-)$ superscript specify that $l_{ \pm}$is for the positively (negatively) charged lepton. Similarly, the CS expression for $\varphi$ in terms of laboratory-frame quantities is given by

$$
\tan \varphi=\frac{\sqrt{M^{2}+p_{\mathrm{T}}^{2}}}{M} \frac{\vec{\Delta} \cdot \hat{R}_{\mathrm{T}}}{\vec{\Delta} \cdot \hat{p}_{\mathrm{T}}},
$$

where $\vec{\Delta}$ is the difference between the $\ell^{-}$and $\ell^{+}$laboratoryframe momentum vectors; $\hat{R}_{\mathrm{T}}$ is the unit vector along $\vec{p}_{p} \times \vec{p}$, with $\vec{p}_{p}$ being the proton momentum vector and $\vec{p}$ the lepton-pair momentum vector; and $\hat{p}_{\mathrm{T}}$ is the unit vector along the transverse component of the lepton-pair momentum vector. At $p_{\mathrm{T}}=0$, the angular distribution is azimuthally symmetric. The right-hand sides of the definitions of $\cos \vartheta$ and $\tan \varphi$ are invariant under Lorentz boosts along the $z$ direction in the laboratory frame.

The angular distribution of Drell-Yan lepton pairs is defined as the ratio of the production cross section to the angle-integrated production cross section. Its general structure consists of terms derived from nine helicity cross sections that describe the polarization state of the boson $[18,19]$,

$$
\begin{aligned}
\frac{d N}{d \Omega} \propto & \left(1+\cos ^{2} \vartheta\right)+A_{0} \frac{1}{2}\left(1-3 \cos ^{2} \vartheta\right)+A_{1} \sin 2 \vartheta \cos \varphi \\
& +A_{2} \frac{1}{2} \sin ^{2} \vartheta \cos 2 \varphi+A_{3} \sin \vartheta \cos \varphi+A_{4} \cos \vartheta \\
& +A_{5} \sin ^{2} \vartheta \sin 2 \varphi+A_{6} \sin 2 \vartheta \sin \varphi+A_{7} \sin \vartheta \sin \varphi
\end{aligned}
$$

The coefficients $A_{0-7}$ are functions of kinematic variables of the boson and vanish when the lepton-pair transverse momentum approaches zero, except for $A_{4}$, which contributes to the tree-level amplitude and generates the forward-backward asymmetry in $\cos \vartheta$. Thus, at zero transverse momentum, the angular distribution reduces to the tree-level form $1+\cos ^{2} \vartheta+A_{4} \cos \vartheta$. In the CS frame, the $A_{0}, A_{2}$, and $A_{4}$ coefficients are large relative to the other coefficients.

The $A_{4} \cos \vartheta$ term violates parity conservation, and is due to the interference of the amplitudes of the vector and axial-vector currents. Its presence induces an asymmetry in the $\varphi$-integrated $\cos \vartheta$ dependence of the cross section. Two sources contribute: the interference between the Z-boson vector and axial-vector amplitudes, and the interference between the photon vector and $Z$-boson axial-vector amplitudes. The asymmetry component from the $\gamma^{*}-Z$ interference cross section depends on axial-vector couplings $g_{A}^{f}$ to fermions $f$ that are independent of $\sin ^{2} \theta_{W}$. The asymmetry component from $Z$-boson selfinterference depends on a product of $g_{V}^{\ell}$ and $g_{V}^{q}$ from the lepton and quark vertices, and thus is related to $\sin ^{2} \theta_{W}$. At the Born level, this product is

$$
T_{3}^{\ell}\left(1-4\left|Q_{\ell}\right| \sin ^{2} \theta_{W}\right) T_{3}^{q}\left(1-4\left|Q_{q}\right| \sin ^{2} \theta_{W}\right),
$$

where $\ell$ and $q$ denote the lepton and quark, respectively. For the Drell-Yan process, the relevant quarks are predominantly the light quarks $u, d$, and $s$. The coupling factor has an enhanced sensitivity to $\sin ^{2} \theta_{W}$ at the lepton- $Z$ vertex: for a $\sin ^{2} \theta_{W}$ value of 0.223 , a $1 \%$ variation in $\sin ^{2} \theta_{W}$ changes the lepton factor $\left(1-4\left|Q_{\ell}\right| \sin ^{2} \theta_{W}\right)$ by about $8 \%$, and it changes the quark factor $\left(1-4\left|Q_{q}\right| \sin ^{2} \theta_{W}\right)$ by about $1.5 \%(0.4 \%)$ for the $u$ ( $d$ or $s$ ) quark. Electroweak radiative corrections do not significantly alter this Born-level interpretation. Loop and vertex electroweak radiative corrections induce multiplicative form-factor corrections [20-22] to the $T_{3}^{f}$ and $\sin ^{2} \theta_{W}$ terms that change their values by a few percent [7].

The forward-backward asymmetry of the polar-angle distribution is defined as

$$
A_{\mathrm{fb}}(M)=\frac{\sigma_{\mathrm{f}}(M)-\sigma_{\mathrm{b}}(M)}{\sigma_{\mathrm{f}}(M)+\sigma_{\mathrm{b}}(M)}=\frac{3}{8} A_{4}(M),
$$

where $\sigma_{\mathrm{f}}$ is the Drell-Yan cross section for the forward (f) orientation of lepton pairs, $\cos \vartheta \geq 0$, and $\sigma_{\mathrm{b}}$ is for the backward (b) orientation of lepton pairs, $\cos \vartheta<0$. Figure 2 shows the typical dependence of the asymmetry

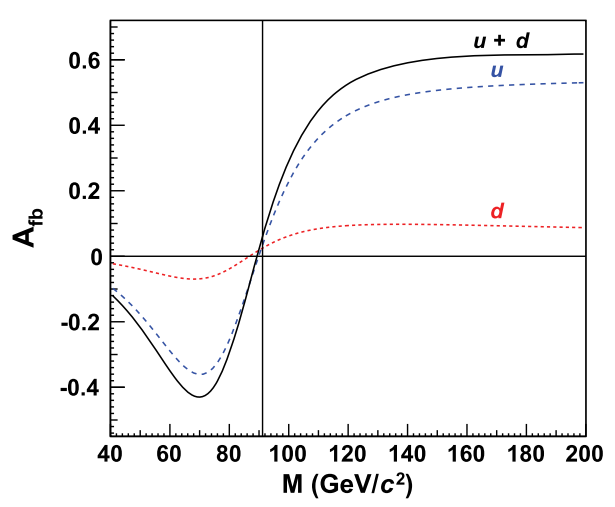

FIG. 2. Typical dependence of $A_{\mathrm{fb}}$ as a function of the leptonpair invariant mass $M$. The vertical line is at $M=M_{Z}$. The label $u+d$ denotes the overall asymmetry, and the labels $u$ and $d$ denote the contribution to the overall asymmetry from quarks with charge $2 / 3$ and $-1 / 3$, respectively. The asymmetry identified by the $u$ or $d$ label is defined as $\left(\sigma_{\mathrm{f}}^{q}-\sigma_{\mathrm{b}}^{q}\right) / \sigma$, where $q=u$ or $d, \sigma_{\mathrm{f}(\mathrm{b})}$ is the forward (backward) cross section, and $\sigma$ is the corresponding cross section from quarks of all charges. Thus, the overall asymmetry is the sum of the asymmetries identified by the $u$ and $d$ labels. 
as a function of the lepton-pair invariant mass from a DrellYan quantum chromodynamics calculation. The offset of $A_{\mathrm{fb}}$ from zero at $M=M_{Z}$ is related to $\sin ^{2} \theta_{W}$. At leptonpair masses much smaller or larger than the $Z$-pole mass, the asymmetry is dominated by the component from $\gamma^{*}-Z$ interference, whose cross section contains the factor $\left(M^{2}-M_{Z}^{2}\right) / M^{2}$ from the photon and $Z$-boson propagators, the factor for the flux of quarks from the parton distribution functions (PDF) of the proton, and the factor for the coupling term $4 / 9(1 / 9)$ at the quark-photon vertex for the charge $2 / 3(-1 / 3)$ quark. Consequently, the asymmetry distribution is sensitive to both $\sin ^{2} \theta_{W}$ and the PDF of the proton.

\section{Extraction of $\sin ^{2} \theta_{\text {eff }}^{\text {lept }}$}

The $\sin ^{2} \theta_{\text {eff }}^{\text {lept }}$ parameter is derived from comparisons of the measurement of $A_{\mathrm{fb}}(M)$ in bins of mass covering the measurement range, and predictions of the measurement (templates) for various input values of the effective-leptonic parameter. The value that minimizes the $\chi^{2}$ between the measurement and templates is the extracted value of $\sin ^{2} \theta_{\text {eff }}^{\text {lept }}$. Detector effects that bias the asymmetry measurement are removed from the measurement by CDF, and incorporated into the templates by $\mathrm{D} 0$. In both the CDF and D0 analyses, the forward-backward asymmetries of electron and muon pairs are separately measured.

The CDF and D0 measurements are based on multiple analyses with improvements over time. Different PDFs have been utilized by CDF and D0, as well as slightly different electroweak correction techniques based on the $\mathrm{SM}$ in the extraction of the $\sin ^{2} \theta_{\mathrm{eff}}^{\text {lept }}$ parameter. For the combination of electron- and muon-channel results within an experiment, and for the Tevatron combination, a framework with two common components, denoted as the "common framework," is adopted to obtain consistent results for the effective-leptonic parameter. The common components are electroweak-radiative corrections calculated using the SM software package ZFITTER 6.43 [20-22], which is used with LEP-1 and SLD measurement inputs for precision tests of the $S M$ at the $Z$ pole [4,5], and NNPDF 3.0 [23-29] PDFs, which include new HERA and LHC data, and replace the Tevatron $W$-asymmetry measurements with lepton-asymmetry measurements from the LHC. A summary of the CDF and D0 measurements is presented in Sec. II, along with the strategies used to achieve results consistent with the common-framework agreement. The combination of these CDF and D0 results is presented in Secs. III, and IV provides the summary.

\section{INPUT MEASUREMENTS}

The Fermilab Tevatron collider provides $p \bar{p}$ collisions at a center-of-momentum energy of $1.96 \mathrm{TeV}$. The CDF [30] and D0 [31] detectors are general-purpose detectors that surround the Tevatron collision regions. For both detectors, precision charged-particle tracking detectors (trackers) immersed in solenoidal magnetic fields are located around the beam line. Beyond the trackers are projective-tower electromagnetic and hadronic calorimeters with transverse and longitudinal segmentation, which are used for the identification of electrons, photons, and clusters of outgoing particles (jets), and for measurements of their kinematic properties. The outer regions of each detector consist of hadron absorbers and trackers for the detection and identification of muons.

For particle trajectories, the polar and azimuthal angles of the coordinate systems are denoted by $\theta_{\text {lab }}$ and $\phi_{\text {lab }}$ respectively. The pseudorapidity of a particle is $\eta=$ $-\ln \tan \left(\theta_{\text {lab }} / 2\right)$. Detector coordinates are specified as $\left(\eta_{\text {det }}, \phi_{\text {det }}\right)$, where $\eta_{\text {det }}$ is the pseudorapidity relative to the detector center $\left(z_{\mathrm{lab}}=0\right)$. For particle energies measured in the calorimeters, the transverse energy $E_{\mathrm{T}}$ is defined as $E_{\mathrm{T}}=E \sin \theta_{\text {lab }}$.

Both the CDF and D0 measurements utilize electron and muon pairs reconstructed in high- $p_{\mathrm{T}}$ electron and muon data samples, respectively. During data taking, events with high- $p_{\mathrm{T}}$ electrons and muons are selected online by triggers. Offline selection criteria are applied to these samples to improve the quality of the sample used for the asymmetry measurements.

\section{A. CDF measurement}

The CDF measurements for the electron [9] and muon [8] channels use the full Tevatron Run II data set, corresponding to an integrated luminosity of $9 \mathrm{fb}^{-1}$ of $p \bar{p}$ collisions. Similar methods are used for both analyses. The $A_{\mathrm{fb}}$ measurements are corrected for detector effects. The effects of detector resolution and quantum electrodynamics (QED) final-state radiation (FSR) are removed from the measurements using the CDF simulation of Drell-Yan events. The templates are strictly quantum chromodynamics (QCD) calculations of $A_{\mathrm{fb}}$.

Approximately 485000 electron pairs and 277000 muon pairs are utilized in the measurements. Details of the electron- and muon-selection criteria are presented in Refs. $[9,8]$, respectively. Kinematic, fiducial, and leptonidentification criteria are applied. As Drell-Yan leptons are usually produced in isolation from the other activity in the event, isolation criteria that limit event activity around the leptons are also applied. In the electron channel, one electron must be detected in the central-calorimeter region $0.05<\left|\eta_{\text {det }}\right|<1.05$, and its partner electron can be either in the central-calorimeter region or the plug-calorimeter region $1.2<\left|\eta_{\text {det }}\right|<2$.8. For central-central electron pairs, one electron is required to have $E_{\mathrm{T}}>25 \mathrm{GeV}$ and the other to have $E_{\mathrm{T}}>15 \mathrm{GeV}$. For central-plug electron pairs, both electrons are required to have $E_{\mathrm{T}}>20 \mathrm{GeV}$. In the muon channel, the selection requires both muons to be predominantly within the central muon-detector region $\left|\eta_{\text {det }}\right|<1$, and have $p_{\mathrm{T}}>20 \mathrm{GeV} / c$. For the asymmetry 
measurements, electron and muon pairs are selected to have invariant masses in the range $50-350 \mathrm{GeV} / \mathrm{c}^{2}$ and $50-1000 \mathrm{GeV} / c^{2}$, respectively. The upper limit for muon pairs is larger to accommodate the significant resolution smearing of the muon momentum at high masses.

The $A_{\mathrm{fb}}$ measurement uses a data-driven event-weighting method [32], which is equivalent to performing individual measurements of the asymmetry in $|\cos \vartheta|$ bins and then combining them. The standard expression for the asymmetry in a bin is

$$
A_{\mathrm{fb}}=\frac{N_{\mathrm{f}} /(\epsilon A)_{\mathrm{f}}-N_{\mathrm{b}} /(\epsilon A)_{\mathrm{b}}}{N_{\mathrm{f}} /(\epsilon A)_{\mathrm{f}}+N_{\mathrm{b}} /(\epsilon A)_{\mathrm{b}}},
$$

where $N_{\mathrm{f}(\mathrm{b})}$ and $(\epsilon A)_{\mathrm{f}(\mathrm{b})}$ are the signal event counts, and the efficiency $(\epsilon)$ and acceptance $(A)$ product, respectively, of forward (backward) lepton pairs. Only leptons reconstructed within the central region, where tracking performance is optimal with small charge misidentification probability, are used to determine the forward (backward) orientation of a pair. For these leptons, the track-finding efficiency is $99 \%$ [33]. Within a localized $|\cos \vartheta|$ bin, the forward and backward dependence of the acceptance and efficiency of its lepton pairs cancels out to first order so that

$$
A_{\mathrm{fb}} \approx \frac{N_{\mathrm{f}}-N_{\mathrm{b}}}{N_{\mathrm{f}}+N_{\mathrm{b}}} .
$$

The similarity of $(\epsilon A)_{\mathrm{f}}$ and $(\epsilon A)_{\mathrm{b}}$ for a value of $|\cos \vartheta|$ is related to the proximity of lepton-pair trajectories within the detector to those of the ones with the charges exchanged. With the exchange, the magnitude of $\cos \vartheta$ is unchanged but the sign is reversed, i.e., forwards and backwards are interchanged. The exchange transforms a lepton with charge $Q$ into one with charge $-Q$ but leaves its laboratory momentum $\vec{p}$ unchanged. For a typical lepton with $p_{\mathrm{T}} \sim 40 \mathrm{GeV} / c$, the radius of curvature in the magnetic-field-bend plane is $100 \mathrm{~m}$. For reference, the radial extent of the precision trackers in this plane is $1.3 \mathrm{~m}$. Thus, the trajectories of a lepton and its charge-exchanged configuration through the detector are localized, so to first order, their probabilities of being reconstructed and selected are the same.

The expected angular dependencies of the numeratorevent difference and denominator-event sum of Eq. (9) are derived using Eq. (5). The numerator difference is proportional to $2 A_{4}|\cos \vartheta|$, and the denominator sum to $2\left(1+\cos ^{2} \vartheta+\cdots\right)$, where $1+\cos ^{2} \vartheta+\cdots$ denotes the $\cos \vartheta$ symmetric terms from Eq. (5). Together they yield $A_{\mathrm{fb}}=A_{4} \xi$, where $\xi=|\cos \vartheta| /\left(1+\cos ^{2} \vartheta+\cdots\right)$. Each bin is an independent measurement of $A_{4}$, with an uncertainty of $\sigma / \xi$, where $\sigma$ is the statistical uncertainty of $A_{\mathrm{fb}}$. When the measurements are combined, each bin has a statistical weight of $\sigma^{-2}$ and a sampling weight of $\xi^{2}$. The binned measurements are reformulated into an unbinned-asymmetry expression of the form shown in the right-hand side of
Eq. (9) using event weights. Weights for individual events in the numerator and denominator remove the angular dependencies of the event difference and sum, respectively, and provide the sampling weight for the combination of events across $|\cos \vartheta|$.

Because the event-weighting method needs events for corrections, kinematic regions with few or no events are eliminated from the acceptance region of the measurement and template calculations. Consequently, the kinematicacceptance region of the electron-pair is restricted to $|y|<1.7$, and that of the muon-pair to $|y|<1$. Small secondary effects not corrected by the event-weighting method are removed with the simulation.

The simulation of Drell-Yan events, described below, uses PYTHIA 6.2 [34] with CTEQ5L [35] PDFs to generate events and includes QED FSR for the decay leptons. To account for QED FSR from promptly decaying hadrons and their decay products, the event generation then uses PнотоS 2.0 [36-38]. This is followed by the detector simulation based on GEANT-3 and GFLASH [39]. Some of the kinematic distributions of the generated $\gamma^{*} / Z$ boson are adjusted with event weights for better agreement between the simulation and the data. For the electron channel, simulation adjustments cover all aspects of boson production because of the extended boson-rapidity coverage of the measurement. The generated boson kinematics are adjusted using the data and the RESBOS [40-43] calculation with CTEQ6.6 [44] PDFs. The generator-level $p_{\mathrm{T}}$ distribution of the boson is adjusted so that the reconstructed $p_{\mathrm{T}}$ distribution of simulated events matches the distribution of the data in two rapidity bins, $0<|y|<0.8$ and $|y| \geq 0.8$. The generator-level bosonmass distribution is adjusted with a mass-dependent factor, which is the ratio of the RESBOS boson-mass distribution calculated using CTEQ6.6 PDFs relative to the PYTHIA 6.4 [45] distribution calculated using CTEQ5L PDFs. For the muon channel, the generator-level $p_{\mathrm{T}}$ distribution of the boson is adjusted so that the reconstructed $p_{\mathrm{T}}$ distribution of simulated events matches the data.

The energy scales of both the data and simulation are calibrated to a common standard following Ref. [46]. The standard consists of idealized calibration distributions derived from the same generated-event samples of the simulation using a detector with perfect calibrations but with the observed resolutions. The energy resolution in the simulation is calibrated to that of the data. In addition to the generator level tuning, other distributions such as the timedependent Tevatron beam-luminosity profile and detector responses near boundaries are tuned. Response adjustments are consistently applied to parity-symmetric variables such as $\left|\eta_{\operatorname{det}}\right|$ or $|\cos \vartheta|$. They are needed for an unbiased detector-resolution unfolding of the asymmetry distribution in mass and $\cos \vartheta$ for the data. Unfolding matrices are used, and the unfolding statistically removes the effects of resolution smearing and QED FSR. The simulation is also used to derive the error matrix for the $A_{\mathrm{fb}}$ measurement. 
The backgrounds are from the production of QCD dijets, $W+$ jets, $\gamma^{*} / Z \rightarrow \tau \tau$, diboson ( $W W, W Z$, and $Z Z$ ), and $t \bar{t}$ events. QCD dijet backgrounds are estimated using the data. Other backgrounds listed above are estimated with PYTHIA 6.2 [34]. For the electron channel, the overall background level amounts to $1.1 \%$ over the mass range of the asymmetry measurement. For the muon channel, the overall background level amounts to $0.5 \%$ over the mass range of the asymmetry measurement. All backgrounds are subtracted from the data.

The CDF templates for $A_{\mathrm{fb}}$ which are compliant with the specifications of the common framework are denoted as "common-framework compliant templates". They are calculated using the POWHEG-BOX next-to-leading-order (NLO) implementation [47] of the Drell-Yan process [48] followed by PYTHIA 6.41 [45] parton-showering. The combined implementation has next-to-leading-log resummation accuracy. The NNPDF 3.0 next-to-next-to-leading order (NNLO) PDFs are used for the parton fluxes.

The complex-valued ZFITTER form factors are incorporated into the POWHEG-BOX amplitudes as specified in the Appendix. The QED photon propagator correction from fermion loops is also included. The implementation of these form factors provides an enhanced Born approximation (EBA) to the electroweak couplings. For consistency with the ZFITTER calculations, the NNPDFs selected are derived assuming a value of the strong-interaction coupling of 0.118 at the $Z$-boson mass. With ZFITTER corrections, the electroweak mixing parameter for the templates is the static on-shell $\sin ^{2} \theta_{W}$. The asymmetry is directly sensitive to the effective mixing terms, which are provided by ZFITTER as $\kappa_{f} \sin ^{2} \theta_{W}$, where $\kappa_{f}$ denotes a fermion-flavor $(f)$ dependent form factor. Unlike the directly observable effective mixing terms, $\sin ^{2} \theta_{W}$ and $\kappa_{f}$ are inferred in the context of the SM and their inputs are specified in the Appendix. The effective mixing terms are functions of $\sin ^{2} \theta_{W}$ and a mass scale. For comparisons with other measurements, the value of the $\sin ^{2} \theta_{\text {eff }}^{\text {lept }}$ parameter is defined at the $Z$ pole and its value is $\operatorname{Re}\left[\kappa_{e}\left(\sin ^{2} \theta_{W}, M_{Z}^{2}\right)\right] \sin ^{2} \theta_{W}$.

The NNPDF 3.0 parton distributions consist of an ensemble of 100 equally probable PDFs for which the value of a calculation is the average of the calculations over the ensemble, and the rms about the average is the PDF uncertainty. A measurement can be incorporated into the ensemble of a PDF fit without regenerating the ensemble via various Bayesian methods that reweight each ensemble PDF [49]. With the Giele-Keller (GK) method, the ensemble PDFs, numbered 1 to $N$, are reweighted with the likelihood of the prediction using the ensemble PDF relative to the measurement,

$$
w_{k}=\frac{\exp \left(-\frac{1}{2} \chi_{k}^{2}\right)}{\sum_{l=1}^{N} \exp \left(-\frac{1}{2} \chi_{l}^{2}\right)},
$$

where $w_{k}$ is the weight for PDF number $k$, and $\chi_{k}^{2}$ is the $\chi^{2}$ between the measurement and the prediction using that PDF [50,51].

For the extraction of the $\sin ^{2} \theta_{\text {eff }}^{\text {lept }}$ parameter, commonframework compliant templates with varying values of the effective-leptonic parameter are calculated for each of the ensemble PDFs of NNPDF 3.0, and the GK-weighting method is used to evaluate the ensemble average and rms of the effective-leptonic parameter derived from the measurement and templates. Consequently, additional PDF constraints from the asymmetry measurement are incorporated into the ensemble of PDFs.

The electron-channel measurement of the asymmetry, along with common-framework compliant templates and the error matrix of the measurement, are used to extract the $\sin ^{2} \theta_{\text {eff }}^{\text {lept }}$ parameter. The result for the electron channel is

$$
\begin{aligned}
\sin ^{2} \theta_{\text {eff }}^{\text {lept }}= & 0.23248 \pm 0.00049 \quad \text { (stat) } \\
& \pm 0.00004 \quad \text { (syst) } \\
& \pm 0.00019 \quad \text { (PDF). }
\end{aligned}
$$

The systematic uncertainty consists of contributions from the energy scale and resolution, the backgrounds, and the QCD scale.

The muon-channel measurement of the asymmetry and the error matrix of the measurement used for measurementto-template $\chi^{2}$ comparisons are presented in Ref. [8]. The published result for the muon channel is

$$
\begin{aligned}
\sin ^{2} \theta_{\text {eff }}^{\text {lept }}= & 0.2315 \pm 0.0009 \quad(\text { stat }) \\
& \pm 0.0002 \quad(\text { syst }) \\
& \pm 0.0004 \quad(\mathrm{PDF}) .
\end{aligned}
$$

The systematic uncertainty consists of contributions from the energy scale and resolution, the backgrounds, and the higher-order terms of QCD. The $A_{\mathrm{fb}}$ templates used for the derivation of this result are from previous iterations of the analysis and not compliant with the specifications of the common framework. They are calculated with a modified version of RESBOS using CTEQ6.6 PDFs and the ZFITTER form factors. Systematic uncertainties are estimated using the POWHEG-BOX NLO parton generator with CT10 NLO PDFs [52], and followed by PYTHIA 6.41 parton showering. The PDF uncertainty is derived from the CT10 uncertainty PDFs at 68\% C.L. The result shown in Eq. (12) is the standalone result of the muon channel, but the methods used do not facilitate a straightforward combination with the electron-channel analysis.

For the combination of the electron- and muon-channel results, the measured asymmetries are directly utilized. Both the electron- and muon-channel measurements are compared against common-framework compliant templates for the extraction of the $\sin ^{2} \theta_{\text {eff }}^{\text {lept }}$ parameter. For comparison purposes, the extracted value of the effective-leptonic 
TABLE I. Summary of the CDF systematic uncertainties on the electron- and muon-channel combination of the electroweak mixing parameter $\sin ^{2} \theta_{\text {eff }}^{\text {lept }}$. The column labeled $\delta \sin ^{2} \theta_{\text {eff }}^{\text {lept gives }}$ the uncertainty of each source. The values are from Table VI of Ref. [9].

\begin{tabular}{ll}
\hline \hline Source & $\delta \sin ^{2} \theta_{\mathrm{eff}}^{\text {lept }}$ \\
\hline Energy scale and resolution & \pm 0.00002 \\
Backgrounds & \pm 0.00003 \\
QCD scale & \pm 0.00006 \\
NNPDF 3.0 PDF & \pm 0.00016 \\
\hline \hline
\end{tabular}

parameter from the muon-asymmetry measurement in this framework is $0.23141 \pm 0.00086$, where the uncertainty is statistical only [9].

The CDF result combining electron and muon channels presented in Ref. [9] is

$$
\begin{aligned}
\sin ^{2} \theta_{\mathrm{eff}}^{\text {lept }}= & 0.23221 \pm 0.00043 \quad(\text { stat }) \\
& \pm 0.00007 \quad(\text { syst }) \\
& \pm 0.00016 \quad(\mathrm{PDF})
\end{aligned}
$$

The systematic uncertainty, consisting of contributions from the energy scale and resolution, the backgrounds, and the QCD scale, are summarized in Table I. As the $A_{\mathrm{fb}}$ templates for the electron- and muon-channel asymmetries are both calculated with the same common-framework infrastructure, the electron- and muon-channel comparison $\chi^{2}$ 's between the data and templates are combined into a joint $\chi^{2}$ for the determination of the best-fit $\sin ^{2} \theta_{\mathrm{eff}}^{\text {lept }}$. The joint $\chi^{2}$ takes into account correlations between the electron- and muon-channel asymmetries for each of the ensemble PDFs of NNPDF 3.0. The GK-weighting method using the joint $\chi^{2}$ incorporates PDF information from both the electron- and muon-channel asymmetry measurements into the PDF ensemble, and thus reduces the PDF uncertainty relative to the default (equal-weight) ensemble.

\section{B. D0 measurement}

The D0 measurement of $\sin ^{2} \theta_{\mathrm{eff}}^{\text {lept }}$ is performed in the electron [12] and muon [13] channels. The electron- and muon-channel results use $9.7 \mathrm{fb}^{-1}$ and $8.6 \mathrm{fb}^{-1}$ of recorded luminosity, respectively.

The asymmetry $A_{\mathrm{fb}}$ is measured in the electron channel using events with at least two electromagnetic (EM) clusters reconstructed in the calorimeter. They are required to be in the central calorimeter (CC) or end calorimeter (EC) with transverse momentum $p_{\mathrm{T}}>25 \mathrm{GeV} / c$. Clusters in the $\mathrm{CC}$ must be matched to reconstructed tracks. For events with both clusters in the EC, only one cluster must be trackmatched. Compared to previous D0 results based on $1.1 \mathrm{fb}^{-1}$ and $5.0 \mathrm{fb}^{-1}$ of luminosity $[10,11]$, the acceptance is extended from $\left|\eta_{\text {det }}\right|<1.0$ to $\left|\eta_{\text {det }}\right|<1.1$ for $\mathrm{CC}$ and from $1.5<\left|\eta_{\text {det }}\right|<2.5$ to $1.5<\left|\eta_{\text {det }}\right|<3.2$ for EC, and previously rejected electrons reconstructed near azimuthal $\mathrm{CC}$ module boundaries are included. By extending the $\eta_{\text {det }}$ and module boundary acceptance, a $70 \%$ increase is achieved in the number of sample events above what would be expected from the increase in luminosity to the full data set, for a total of approximately 560000 electron pairs in the final sample. Events are categorized as CC-CC, CC-EC, or EC-EC based on the $\eta_{\text {det }}$ regions of the two electron candidates.

Muon-channel events are required to have at least two muon candidates reconstructed in the tracking and muon systems, with transverse momenta $p_{\mathrm{T}}>15 \mathrm{GeV} / c$. Both muon candidates are required to have $\left|\eta_{\text {det }}\right|<1.8$ with at least one muon within $\left|\eta_{\operatorname{det}}\right|<1.6$, and they must have tracks matched in the tracking and muon systems. Tracks are required to have opposite curvature. Events with muons nearly back-to-back are removed to reduce cosmic-ray background. The large kinematic acceptance yields a final sample consisting of approximately 481000 muon pairs.

Simulated Drell-Yan events are generated using leading-order PYTHIA 6.23 [34] with the NNPDF 2.3 [24] PDFs for the electron channel and NNPDF 3.0 PDFs for the muon channel, followed by a GEANT-3-based simulation [53] of the D0 detector. The inner-tracker solenoid and muon system toroid polarities are reversed every two weeks on average at D0, enabling cross-checks and cancellations of charge-dependent asymmetries. For example, the muon Drell-Yan samples are generated with different polarities of the solenoid and toroid magnetic fields in the GEANT-3 simulation, and used to model the data corresponding to each combination separately. Data and simulation samples corresponding to different solenoid and toroid polarities are weighted to correspond to equal luminosity exposures for each solenoid-toroid polarity combination. This weighted combination provides cancellation of asymmetries due to variations in detector response. The same reconstruction algorithm is used for the data and simulation.

Relative to previously reported results, new methods of electron energy and muon momentum calibration are developed and applied to both data and simulation. In addition to scale factors, offset parameters are applied to the electron energy as functions of $\eta_{\text {det }}$ and instantaneous luminosity. For muon momentum, a scale factor is introduced that is dependent on charge, $\eta_{\text {det }}$, and solenoid polarity. With these calibration methods, the systematic uncertainties due to energy and momentum modeling are reduced to negligible levels.

The backgrounds are from the production of multijets, $W+$ jets, $\gamma^{*} / Z \rightarrow \tau \tau$, dibosons ( $W W$ and $W Z$ ), and $t \bar{t}$ events. The multijet backgrounds are estimated using the data. The $W+$ jets events are generated using ALPGEN [54] interfaced to PYTHIA 6.23 for showering and hadronization. The other backgrounds are estimated using the PYTHIA 6.23 simulations. At the Z-boson mass peak, the overall 
background level is $0.35 \%(0.88 \%)$ with respect to the total number of selected dielectron (dimuon) events in the data.

The $A_{\mathrm{fb}}$ templates are calculated using PYTHIA 6.23 with NNPDF 2.3 (electron channel) and NNPDF 3.0 (muon channel). They are then reweighted to incorporate higherorder QCD effects. The Z-boson distribution as a function of rapidity and transverse momentum $\left(y, p_{\mathrm{T}}\right)$ is reweighted in both variables to match that from RESBOS [40-43] with CTEQ6.6 [44] PDFs. The boson-mass distribution is reweighted with a mass-dependent NNLO K-factor [55]. The events are processed by the D0 detector simulation to yield templates that include detector resolution effects.

The $A_{\mathrm{fb}}$ distributions in data are obtained as a function of the dilepton invariant mass. For the electron channel, this is done separately for CC-CC, CC-EC, and EC-EC event categories. The weak mixing parameter is extracted from the background-subtracted $A_{\mathrm{fb}}$ spectrum in the regions $75<M_{e e}<115 \mathrm{GeV} / c^{2}$ for CC-CC and CC-EC events, $81<M_{e e}<97 \mathrm{GeV} / c^{2}$ for EC-EC events, and $74<$ $M_{\mu \mu}<110 \mathrm{GeV} / c^{2}$ for muon events, by comparing the data to simulated $A_{\mathrm{fb}}$ templates corresponding to different input values of $\sin ^{2} \theta_{W}$.

Combining the weak-mixing-parameter results from the three electron event categories gives the electron-channel result

$$
\begin{aligned}
\sin ^{2} \theta_{\text {eff }}^{\text {meas }}= & 0.23139 \pm 0.00043 \quad \text { (stat }) \\
& \pm 0.00008 \quad \text { (syst) } \\
& \pm 0.00017 \quad(\mathrm{PDF}) .
\end{aligned}
$$

The sources of systematic uncertainties include energy calibration, energy resolution smearing, backgrounds, charge misidentification, and electron identification [12]. The largest component is from the electron identification (0.00007). The PDF uncertainty is obtained using the equally probable ensemble PDFs of NNPDF 2.3 following the method prescribed by the NNPDF group [24].

The extraction of the weak mixing parameter from the muon channel $A_{\mathrm{fb}}$ distribution in data gives

$$
\begin{aligned}
\sin ^{2} \theta_{\text {eff }}^{\text {meas }}= & 0.22994 \pm 0.00059 \\
& \pm 0.00005 \quad(\text { syst }) \\
& \pm 0.00024 \quad \text { (PDF) }
\end{aligned}
$$

The systematic uncertainties include the following sources: momentum calibration, momentum resolution smearing, backgrounds, and muon identification [13]. The PDF uncertainty is obtained using the 100 equally probable ensemble PDFs of NNPDF 3.0 following the method prescribed by the NNPDF group [23].

Corrections are applied to the values of $\sin ^{2} \theta_{\text {eff }}^{\text {meas }}$ in Eqs. (14) and (15) to make them compliant with the agreed-upon common framework using the CDF EBA electroweak-radiative correction implementation, and NNPDF 3.0.

The $A_{\mathrm{fb}}$ templates used by D0 for both the electron- and muon-channel analyses are calculated with PYTHIA, which uses the same fixed value for the effective mixing terms $\sin ^{2} \theta_{\text {eff }}$ of all fermions. The EBA implementation incorporates ZFITTER weak-interaction corrections and the fermion-loop correction to the photon propagator, both of which are complex valued and mass-scale dependent. The effect of using a fixed and constant value for all of the effective mixing terms is investigated by setting all weak form factors to unity so that the effective mixing terms for the templates become $\sin ^{2} \theta_{\text {eff }}^{\text {lept }}$, where only the real part of the photon propagator correction, the running $\alpha_{\mathrm{em}}$, is retained. This implementation, denoted as nonEBA, is the analog to the PYTHIA calculation. The difference,

$\Delta \sin ^{2} \theta_{\text {eff }}^{\text {lept }}($ ZFITTER $)=\sin ^{2} \theta_{\text {eff }}^{\text {lept }}($ EBA $)-\sin ^{2} \theta_{\text {eff }}^{\text {lept }}($ nonEBA $)$,

provides the correction to the values of $\sin ^{2} \theta_{\text {eff }}^{\text {lept }}$ derived using PYTHIA templates, which is needed to convert them to the values derived using ZFITTER form factors.

The difference is calculated using the combination of the CDF electron- and muon-channel $A_{\mathrm{fb}}$ measurements. Both nonEBA and EBA template calculations use NNPDF 3.0 NNLO with $\alpha_{s}\left(M_{Z}\right)=0.118$. Each template contains about $10^{9}$ generated events, and the uncertainty in $A_{\mathrm{fb}}$ for the mass bin containing the $Z$-boson mass is about $5 \times 10^{-5}$. Differences are calculated for 23 ensemble PDFs whose best-fit mixing parameter value from EBA templates is near the average value derived over all ensemble PDFs. The GK-weighted average and rms of $\Delta \sin ^{2} \theta_{\text {eff }}^{\text {lept }}($ ZFITTER) over the 23 PDFs are 0.00022 and 0.00002 , respectively. To accommodate the statistical uncertainty of the effective-leptonic parameter, an additional uncertainty of 0.00003 is assigned, resulting in a total (EBA-nonEBA) uncertainty of \pm 0.00004 . This value of $+0.00022 \pm 0.00004$ is the correction from the PYTHIA framework, which assumes a single value for all the effective mixing parameters, to the ZFITTER-based one.

The D0 electron-channel $A_{\mathrm{fb}}$ template calculations use NNPDF 2.3. Therefore, Eq. (14) must be corrected to comply with the common framework choice of NNPDF 3.0. To calculate the correction, the value of $\sin ^{2} \theta_{\text {eff }}^{\text {lept }}$ is extracted using a default NNPDF 3.0 template and multiple NNPDF 2.3 templates. A PYTHIA template of $5 \times 10^{8}$ events is generated, using NNPDF 3.0 with fixed $\sin ^{2} \theta_{W}$ input, and taken as pseudodata after applying fast-simulation kinematic requirements. In addition, 40 templates of $3 \times 10^{8}$ events each are generated, using NNPDF 2.3 with varying $\sin ^{2} \theta_{W}$ inputs and applying the same selection criteria. These templates are used to 
obtain the best $\chi^{2}$ fit for the $\sin ^{2} \theta_{W}$ value. The difference between the input value for NNPDF 3.0 and the value extracted from NNPDF 2.3, denoted by $\Delta \sin ^{2} \theta_{\text {eff }}^{\text {lept }}$ (PDF), is used to provide a correction to the D0 electron-channel value of $\sin ^{2} \theta_{\text {eff }}^{\text {lept }}$ derived using NNPDF 2.3 to that derived using NNPDF 3.0. The value of $\Delta \sin ^{2} \theta_{\text {eff }}^{\text {lept }}($ PDF) based on the D0 analysis is $-0.00024 \pm 0.00004$, where the uncertainty is statistical. A similar calculation in the CDF framework confirms this value.

The uncorrected central value of the D0 electronchannel measurement is 0.23139 [Eq. (14)]. To update this measurement to one based on templates calculated with NNPDF 3.0 PDFs and ZFITTER-based electroweak radiative corrections, the corrections $\Delta \sin ^{2} \theta_{\text {eff }}^{\text {lept }}(\mathrm{PDF})$, and $\Delta \sin ^{2} \theta_{\text {eff }}^{\text {lept }}$ (ZFITTER) are both applied for a net correction of $-0.00002 \pm 0.00005$, where the uncertainty is denoted as "corrections" in Table II. The corrected value is

$$
\begin{aligned}
\sin ^{2} \theta_{\mathrm{eff}}^{\text {lept }}= & 0.23137 \pm 0.00043 \quad(\text { stat }) \\
& \pm 0.00009 \quad(\text { syst }) \\
& \pm 0.00017 \quad(\mathrm{PDF})
\end{aligned}
$$

which is the D0 electron-channel value used to combine with the D0 muon-channel and CDF results for the final Tevatron combination.

The published D0 electron-channel value of $\sin ^{2} \theta_{\text {eff }}^{\text {lept }}$ in Ref. [12] includes no PDF correction, and only a partial electroweak radiative correction that accounts for differences in the effective couplings to leptons, up-type quarks, and down-type quarks, but not for the complex value and mass-scale dependence of the couplings. Therefore, the fully-corrected Eq. (17) is used for the D0 [13] and Tevatron combinations.

The higher-order weak-interaction radiative correction, $\Delta \sin ^{2} \theta_{\text {eff }}^{\text {lept }}$ (ZFITTER), is applied to the uncorrected muonchannel result, Eq. (15), giving a +0.00022 shift to the measured central value and an additional \pm 0.00004

TABLE II. Summary of the D0 systematic uncertainties on the electron- and muon-channel combination of the electroweak mixing parameter $\sin ^{2} \theta_{\text {eff }}^{\text {lept }}$ from Ref. [13]. The column labeled $\delta \sin ^{2} \theta_{\text {eff }}^{\text {lept }}$ gives the uncertainty of each source.

\begin{tabular}{lc}
\hline \hline Source & $\delta \sin ^{2} \theta_{\mathrm{eff}}^{\text {lept }}$ \\
\hline Energy $\left(e^{+} e^{-}\right)$or momentum $\left(\mu^{+} \mu^{-}\right)$calibration & \pm 0.00001 \\
Energy $\left(e^{+} e^{-}\right)$or momentum $\left(\mu^{+} \mu^{-}\right)$resolution & \pm 0.00002 \\
Backgrounds & \pm 0.00001 \\
Charge misidentification & \pm 0.00002 \\
Lepton identification & \pm 0.00005 \\
Fiducial asymmetry & \pm 0.00001 \\
Corrections (PDF and ZFITTER $)$ & \pm 0.00005 \\
NNPDF 2.3 $\left(e^{+} e^{-}\right)$or NNPDF 3.0 $\left(\mu^{+} \mu^{-}\right)$PDF & \pm 0.00019 \\
\hline \hline
\end{tabular}

systematic uncertainty. Applying this correction, the D0 muon-channel final corrected result [13] is

$$
\begin{aligned}
\sin ^{2} \theta_{\text {eff }}^{\text {lept }}= & 0.23016 \pm 0.00059 \quad \text { (stat) } \\
& \pm 0.00006 \quad \text { (syst) } \\
& \pm 0.00024 \quad(\mathrm{PDF})
\end{aligned}
$$

The D0 combination result [13] for $\sin ^{2} \theta_{\text {eff }}^{\text {lept }}$ is obtained using the corrected electron- and muon-channel results as inputs. The central value and systematic uncertainties are combined using the D0 electron- and muon-channel central values with the inverse of the squares of the measurements' statistical uncertainties as weights. The electron and muon systematic uncertainties are treated as uncorrelated, with the exception of the higher-order radiative correction uncertainty which is treated as $100 \%$ correlated. However, the total uncertainty is marginally affected by this choice, because both the electron- and muon-channel total measurement uncertainties are dominated by their statistical uncertainties.

The correlation of PDF uncertainties between the electron- and muon-channel acceptances cannot be ignored. Instead of estimating the correlation matrix between the electron and muon channels, we directly estimate the combined PDF uncertainty. We first estimate the PDF uncertainty on the $A_{\mathrm{fb}}$ observable averaged over the electron and muon channels. We then scale that uncertainty using the linear relation between $A_{\mathrm{fb}}$ and $\sin ^{2} \theta_{\mathrm{W}}$ estimated in simulated events.

The D0 combination of electron- and muon-channel results [13] is

$$
\begin{aligned}
\sin ^{2} \theta_{\text {eff }}^{\text {lept }}= & 0.23095 \pm 0.00035 \quad \text { (stat) } \\
& \pm 0.00007 \quad \text { (syst) } \\
& \pm 0.00019 \quad(\mathrm{PDF})
\end{aligned}
$$

and a summary of the systematic uncertainties of the combination is presented in Table II.

\section{CDF AND D0 COMBINATION}

The Tevatron combination of the $\sin ^{2} \theta_{\text {eff }}^{\text {lept }}$ parameter uses a single value from each experiment, the combined result of the electron- and muon-channel analyses. The analyses used to derive these values are described in Sec. II A for CDF, and Sec. II B for D0. Inferences of the $\sin ^{2} \theta_{W}\left(M_{W}\right)$ parameter corresponding to the Tevatron-combination value of $\sin ^{2} \theta_{\text {eff }}^{\text {lept }}$ and the CDF and D0 input values are obtained using SM calculations in the on-shell renormalization scheme.

\section{A. Tevatron combination}

The central values of the combined electron- and muonchannel results from each experiment are 
TABLE III. Summary of the uncertainties for the electroweak mixing parameter $\sin ^{2} \theta_{\text {eff }}^{\text {lept }}$ from the Tevatron combination of the $\mathrm{CDF}$ and D0 measurements.

\begin{tabular}{lccc}
\hline \hline & \multicolumn{3}{c}{ Uncertainties on $\sin ^{2} \theta_{\mathrm{eff}}^{\text {lept }}$} \\
\cline { 2 - 4 } Source & CDF inputs & D0 inputs & $\begin{array}{c}\text { Tevatron } \\
\text { combination }\end{array}$ \\
\hline Statistics & \pm 0.00043 & \pm 0.00035 & \pm 0.00027 \\
Uncorrelated syst. & \pm 0.00007 & \pm 0.00007 & \pm 0.00005 \\
PDF & \pm 0.00016 & \pm 0.00019 & \pm 0.00018 \\
\hline \hline
\end{tabular}

$$
\begin{gathered}
\sin ^{2} \theta_{\mathrm{eff}}^{\text {lept }}=0.23221 \pm 0.00043(\mathrm{CDF}), \quad \text { and } \\
\sin ^{2} \theta_{\mathrm{eff}}^{\text {lept }}=0.23095 \pm 0.00035(\mathrm{D} 0)
\end{gathered}
$$

where the uncertainties are statistical only. The systematic uncertainties are summarized in Tables I and II. D0 avoids a QCD scale uncertainty by incorporating NNLO effects into the $A_{\mathrm{fb}}$ templates, while CDF avoids sensitivity to lepton identification and detector asymmetry uncertainties through the use of the event-weighting method described in Sec. II A. The PDF uncertainties are treated as $100 \%$ correlated. All other systematic uncertainties in Tables I and II are treated as uncorrelated for the CDF and D0 combination. There are some correlations from the common PYTHIA-derived backgrounds, but since the overall contribution from the background is small and the detectors are different, the backgrounds are treated as uncorrelated.

The combination of the two inputs with the "best linear unbiased estimate" (BLUE) method [56] yields the Tevatron combination,

$$
\begin{aligned}
\sin ^{2} \theta_{\mathrm{eff}}^{\text {lept }}= & 0.23148 \pm 0.00027 \quad(\text { stat }) \\
& \pm 0.00005 \quad(\text { syst }) \\
& \pm 0.00018 \quad(\mathrm{PDF}) .
\end{aligned}
$$

The total uncertainty is \pm 0.00033 , with Table III summarizing the input-source uncertainties and the corresponding combination uncertainties. The combination weights for the CDF and D0 inputs are 0.42 and 0.58 , respectively, and the $\chi^{2}$ probability for the compatibility of the two input measurements is $2.6 \%$.

\section{B. Inference of $\sin ^{2} \theta_{W}$}

The determination of $\sin ^{2} \theta_{\text {eff }}^{\text {lept }}$ is a direct measurement in that the observed asymmetry is directly sensitive to the effective mixing parameters. In order to obtain $\sin ^{2} \theta_{W}$ and its uncertainty, the relationship

$$
\sin ^{2} \theta_{\mathrm{eff}}^{\text {lept }}=\operatorname{Re}\left[\kappa_{e}\left(\sin ^{2} \theta_{W}, M_{Z}^{2}\right)\right] \sin ^{2} \theta_{W}
$$

and the ZFITTER SM calculation of the form factor $\kappa_{e}$ with a set of input parameters are required. The calculation and parameters specified in the Appendix provide the context for the inference of $\sin ^{2} \theta_{W}$. The calculated value of the form factor, $\operatorname{Re}\left[\kappa_{e}\right]$, is 1.0371 for the value of the effective-leptonic mixing parameter specified in Eq. (22) [7]. The choice of the SM-input parameter value for the top-quark mass affects the value of the form factor, and thus the inference of $\sin ^{2} \theta_{W}$. The uncertainty of the inferred value of $\sin ^{2} \theta_{W}$ due to the uncertainty from the top-quark mass input, $173.2 \pm$ $0.9 \mathrm{GeV} / c^{2}$ [57], is \pm 0.00008 [9]. This uncertainty is denoted as the "form factor" uncertainty in Table IV.

For the CDF measurement, the values of $\sin ^{2} \theta_{\text {eff }}^{\text {lept }}$, $\sin ^{2} \theta_{W}$, and $M_{W}$ based on the combined electron- and muon-channel results are

$$
\begin{gathered}
\sin ^{2} \theta_{\text {eff }}^{\text {lept }}=0.23221 \pm 0.00043 \pm 0.00018 \\
\sin ^{2} \theta_{W}=0.22400 \pm 0.00041 \pm 0.00019 \\
M_{W}=80.328 \pm 0.021 \pm 0.010 \mathrm{GeV} / c^{2},
\end{gathered}
$$

and the D0 values of $\sin ^{2} \theta_{\text {eff }}^{\text {lept }}, \sin ^{2} \theta_{W}$, and $M_{W}$ based on the combined electron- and muon-channel results are

$$
\begin{gathered}
\sin ^{2} \theta_{\text {eff }}^{\text {lept }}=0.23095 \pm 0.00035 \pm 0.00020 \\
\sin ^{2} \theta_{W}=0.22269 \pm 0.00034 \pm 0.00021 \\
M_{W}=80.396 \pm 0.017 \pm 0.011 \mathrm{GeV} / c^{2},
\end{gathered}
$$

where the first contribution to each uncertainty is statistical and the second is systematic. All systematic uncertainties are combined in quadrature. For $\sin ^{2} \theta_{W}\left(M_{W}\right)$, the systematic uncertainties include those propagated from $\sin ^{2} \theta_{\text {eff }}^{\text {lept }}$ and the form-factor uncertainty.

The Tevatron-combination values for $\sin ^{2} \theta_{\mathrm{eff}}^{\text {lept }}, \sin ^{2} \theta_{W}$, and $M_{W}$ are

$$
\begin{aligned}
\sin ^{2} \theta_{\mathrm{eff}}^{\text {lept }} & =0.23148 \pm 0.00027 \pm 0.00018 \\
& =0.23148 \pm 0.00033 \\
\sin ^{2} \theta_{W} & =0.22324 \pm 0.00026 \pm 0.00019 \\
& =0.22324 \pm 0.00033
\end{aligned}
$$

TABLE IV. Summary of uncertainties on the inference of the on-shell electroweak mixing parameter $\sin ^{2} \theta_{W}$ for the Tevatroncombination value of $\sin ^{2} \theta_{\text {eff }}^{\text {lept }}$. The column labeled $\delta \sin ^{2} \theta_{W}$ gives the uncertainty of each source. Except for the uncertainty due to the sample size, all other entries are systematic uncertainties.

\begin{tabular}{lc}
\hline \hline Source & $\delta \sin ^{2} \theta_{W}$ \\
\hline Statistics & \pm 0.00026 \\
Uncorrelated & \pm 0.00005 \\
PDF & \pm 0.00017 \\
Form factor $\left(m_{t}=173.2 \pm 0.9 \mathrm{GeV} / c^{2}\right)$ & \pm 0.00008 \\
\hline \hline
\end{tabular}




$$
\begin{aligned}
M_{W} & =80.367 \pm 0.014 \pm 0.010 \mathrm{GeV} / c^{2} \\
& =80.367 \pm 0.017 \mathrm{GeV} / c^{2},
\end{aligned}
$$

where the first contribution to each uncertainty is statistical and the second is systematic. The total systematic uncertainty is the sum in quadrature of all systematic uncertainties listed in Tables III and IV. The form-factor uncertainty is only included in the systematic uncertainty of $\sin ^{2} \theta_{W}$ and $M_{W}$.

\section{Result comparisons}

The measurements of $\sin ^{2} \theta_{\mathrm{eff}}^{\text {lept }}$ are compared with previous results from the $Z$-boson pole mass region in Fig. 3. The hadron-collider results are based on $A_{\mathrm{fb}}$ measurements. The LEP-1 and SLD results are from the individual asymmetry measurements indicated in the figure.

The $W$-boson mass inference is compared in Fig. 4 with previous direct and indirect measurements. The direct measurements are from the Tevatron and LEP-2 [58]. The indirect measurements from the Tevatron are derived from the CDF and D0 measurements of $A_{\mathrm{fb}}$, and their combination. The indirect measurement of $\sin ^{2} \theta_{W}$ from LEP-1 and SLD, $0.22332 \pm 0.00039$, is from a SM fit to all Z-pole measurements [4,5] described in Appendix F of Ref. [5]. In that fit, the

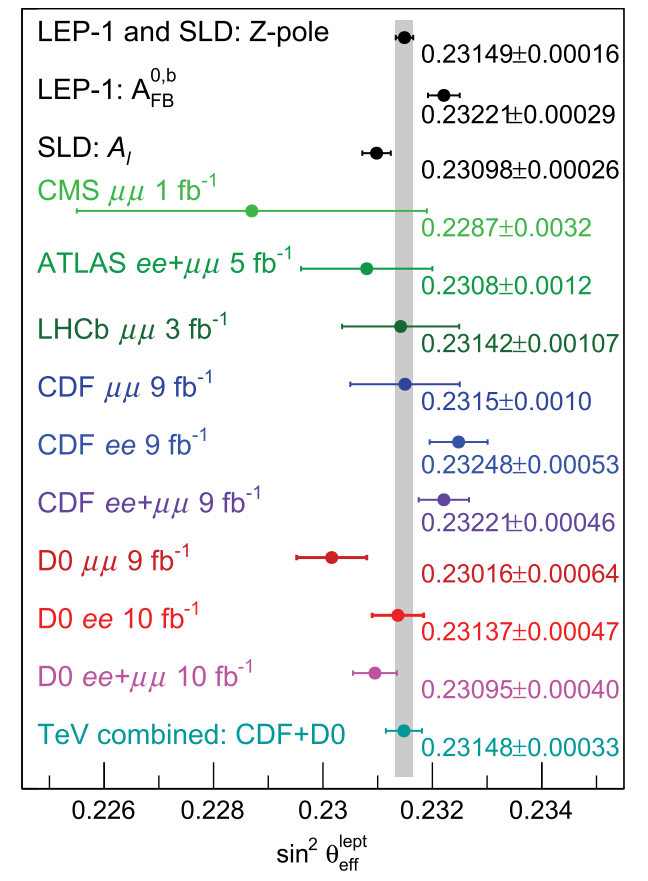

FIG. 3. Comparison of experimental measurements of $\sin ^{2} \theta_{\mathrm{eff}}^{\text {lept }}$ in the region of the $Z$-boson pole mass. The horizontal bars represent total uncertainties. The Tevatron combination (this paper) of CDF and D0 results is denoted as "TeV combined: CDF+D0". The other measurements are from LEP-1 combination [4], SLD [4], CMS [15], ATLAS [14], LHCb [16], CDF [8,9], and D0 [12,13]. The LEP-1 and SLD $Z$ pole result is the combination of their six measurements, and the shaded vertical band shows its uncertainty.

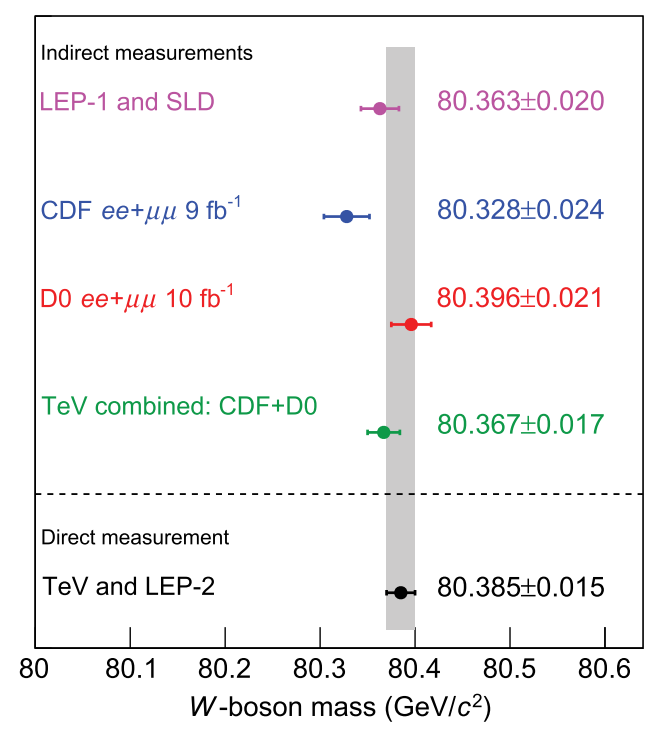

FIG. 4. Comparison of experimental determinations of the $W$-boson mass at high-energy colliders. The horizontal bars represent total uncertainties. The Tevatron combination (this paper) based on $\mathrm{CDF}$ and D0 results is denoted as "TeV combined: CDF + D0". The other indirect measurements are from LEP-1 and SLD [4,5], CDF [8,9], and D0 [12,13]. All indirect measurements use the Tevatron top-quark mass measurement specified in the text [57]. The SM context for the Tevatron inferences is specified in the Appendix, and the SM fit of LEP-1 and SLD is described in Appendix F of Ref. [5]. For the Tevatron inferences of the $W$-boson mass, the SM Higgs-boson mass parameter is fixed, while in the LEP-1 and SLD SM fit, it is a floating parameter. The direct measurements are from the Tevatron and LEP-2 [58], and the shaded vertical band shows its uncertainty.

following input parameters to ZFITTER are varied simultaneously within the constraints of the LEP-1 and SLD data: the Higgs-boson mass $m_{H}$, the $Z$-boson mass $M_{Z}$, the QCD coupling at the $Z$ pole $\alpha_{s}\left(M_{Z}^{2}\right)$, and the QED correction $\Delta \alpha_{\mathrm{em}}^{(5)}\left(M_{Z}^{2}\right)$. The top-quark mass $m_{t}$ is constrained to the value measured directly at the Tevatron, $173.2 \pm$ $0.9 \mathrm{GeV} / c^{2}$ [57]. The precision of the Tevatron indirect measurement almost matches that of the direct measurement combination from the Tevatron and LEP-2.

\section{SUMMARY}

The angular distribution of Drell-Yan lepton pairs provides information on the electroweak mixing parameter $\sin ^{2} \theta_{W}$. The effective-leptonic mixing parameter $\sin ^{2} \theta_{\text {eff }}^{\text {lept }}$ is derived from measurements of the forward-backward asymmetry $A_{\mathrm{fb}}(M)$ in the polar-angle distribution by the CDF and D0 experiments, where $M$ is the lepton-pair effective mass. The measurements are based on the full Tevatron proton-antiproton data sets collected in 2001-2011. The CDF measurement is derived from electron and muon pairs from a $p \bar{p}$ collision sample corresponding to $9 \mathrm{fb}^{-1}$ of 
integrated luminosity, and the D0 measurement is derived from electron and muon pairs from $p \bar{p}$ samples corresponding to integrated luminosities of $9.7 \mathrm{fb}^{-1}$ and $8.6 \mathrm{fb}^{-1}$ respectively.

The Tevatron combination of the CDF and D0 results yields

$$
\sin ^{2} \theta_{\mathrm{eff}}^{\text {lept }}=0.23148 \pm 0.00033
$$

The combined result is consistent with LEP-1 and SLD measurements at the $Z$-boson pole. Based on the SM calculations specified in the Appendix, the inferences of $\sin ^{2} \theta_{W}$ and the $W$-boson mass are

$$
\begin{aligned}
\sin ^{2} \theta_{W} & =0.22324 \pm 0.00033, \quad \text { and } \\
M_{W} & =80.367 \pm 0.017 \mathrm{GeV} / c^{2},
\end{aligned}
$$

respectively. Within the context of the $\mathrm{SM}, \sin ^{2} \theta_{W}$ and the $W$-boson mass are related. Comparisons of the indirect measurements of the $W$-boson mass with those from direct measurements provide powerful tests of the self-consistency of the SM.

The combined result on the effective $\sin ^{2} \theta_{W}$ mixing parameter at the lepton vertex $\sin ^{2} \theta_{\text {eff }}^{\text {lept }}$ is the most precise obtained in hadron collisions. It is consistent with, and approaches in precision, the best measurements from electron-positron colliders. The values of $\sin ^{2} \theta_{\text {eff }}^{\text {lept }}$ from hadron and electron-positron colliders are extracted from a complementary set of processes. At hadron colliders, the partonic processes are $q \bar{q} \rightarrow e^{+} e^{-}$and $\mu^{+} \mu^{-}$, and the forward-backward asymmetry is sensitive to the vertex couplings of the outgoing leptons and the predominantly light quarks from the hadrons. At electron-positron colliders, the processes are either leptonic, i.e. $e^{+} e^{-} \rightarrow e^{+} e^{-}$, $\mu^{+} \mu^{-}$, and $\tau^{+} \tau^{-}$, or mixed, i.e. $e^{+} e^{-} \rightarrow q \bar{q}$. While the asymmetry of a mixed process is analogous to that from hadron collisions, events with $b$ quarks in the final state yield the best experimental precision while those with lighter quarks yield significantly less precision. The result of the Tevatron combination supports the central value of $\sin ^{2} \theta_{\text {eff }}^{\text {lept }}$ derived from the LEP-1 and SLD Z-pole measurements, and the combined values from the Tevatron and from LEP-1 and SLD are nearly identical.

\section{ACKNOWLEDGMENTS}

We thank the staffs at Fermilab and collaborating institutions, and acknowledge support from the Department of Energy and the National Science Foundation (United States of America); the Australian Research Council (Australia); the National Council for the Development of Science and Technology and the Carlos Chagas Filho Foundation for the Support of Research in the
State of Rio de Janeiro (Brazil); the Natural Sciences and Engineering Research Council (Canada); the Chinese Academy of Sciences and the National Natural Science Foundation of China (China); the Administrative Department of Science, Technology and Innovation (Colombia); the Ministry of Education, Youth and Sports (Czech Republic); the Academy of Finland (Finland); the Alternative Energies and Atomic Energy Commission and the National Center for Scientific Research/National Institute of Nuclear and Particle Physics (France); the Bundesministerium für Bildung und Forschung (Federal Ministry of Education and Research) and the Deutsche Forschungsgemeinschaft (German Research Foundation) (Germany); the Department of Atomic Energy and Department of Science and Technology (India); the Science Foundation Ireland (Ireland); Istituto Nazionale di Fisica Nucleare (National Institute for Nuclear Physics) (Italy); the Ministry of Education, Culture, Sports, Science and Technology (Japan); the Korean World Class University Program and the National Research Foundation (Korea); the National Council of Science and Technology (Mexico); the Foundation for Fundamental Research on Matter (The Netherlands); the National Science Council (Republic of China); the Ministry of Education and Science of the Russian Federation, the National Research Center "Kurchatov Institute" of the Russian Federation, and the Russian Foundation for Basic Research (Russia); the Slovak R\&D Agency (Slovakia); the Ministry of Science and Innovation and the Consolider-Ingenio 2010 Program (Spain); the Swedish Research Council (Sweden); the Swiss National Science Foundation (Switzerland); the Ministry of Education and Science of Ukraine (Ukraine). the Science and Technology Facilities Council and The Royal Society (United Kingdom); the A.P. Sloan Foundation (United States of America); and the European Union community Marie Curie Fellowship Contract No. 302103.

\section{APPENDIX: ZFITTER}

The effects of virtual electroweak radiative corrections for the Drell-Yan process are obtained from the $Z$-amplitude form factors for fermion-pair production according to $e^{+} e^{-} \rightarrow Z \rightarrow f \bar{f}$. These form factors are calculated by ZFITTER 6.43 [20-22], which is used with LEP-1, SLD, Tevatron, and LHC measurement inputs for precision tests of the SM $[4,5]$.

The input parameters to the ZFITTER radiative-correction calculation are particle masses, the electromagnetic finestructure constant $\alpha_{\mathrm{em}}$, the Fermi constant $G_{F}$, the stronginteraction coupling at the Z-boson mass $\alpha_{s}\left(M_{Z}^{2}\right)$, and the contribution of the light quarks to the "running" $\alpha_{\mathrm{em}}$ at the $Z$ boson mass $\Delta \alpha_{\mathrm{em}}^{(5)}\left(M_{Z}^{2}\right)$. The scale-dependent couplings are $\alpha_{s}\left(M_{Z}^{2}\right)=0.118 \pm 0.001[59]$ and $\Delta \alpha_{\mathrm{em}}^{(5)}\left(M_{Z}^{2}\right)=0.0275 \pm$ 0.0001 [60]. The mass parameters are $M_{Z}=91.1875 \pm$ $0.0021 \mathrm{GeV} / c^{2} \quad[4,5], \quad m_{t}=173.2 \pm 0.9 \mathrm{GeV} / c^{2} \quad$ (top 
quark) [57], and $m_{H}=125 \mathrm{GeV} / c^{2}$ (Higgs boson). Form factors and the $Z$-boson total decay-width $\Gamma_{Z}$ are calculated. The central values of the parameters provide the context of the ZFITTER SM calculations.

The ZFITTER package uses the on-shell renormalization scheme [17], where particle masses are on-shell and $\sin ^{2} \theta_{W}=1-M_{W}^{2} / M_{Z}^{2}$ holds to all orders of perturbation theory by definition. If both $G_{F}$ and $m_{H}$ are specified, $\sin \theta_{W}$ is not independent, and is related to $G_{F}$ and $m_{H}$ by SM constraints from radiative corrections. To vary the value of the $\sin \theta_{W}\left(M_{W}\right)$ parameter, the value of $G_{F}$ is not constrained. The $M_{W}$ value is varied in the range $80.0-80.5 \mathrm{GeV} / c^{2}$, and for each value, ZFITTER calculates $G_{F}$ and the form factors. Each set of calculations corresponds to a family of physics models with SM-like couplings where the parameter $\sin ^{2} \theta_{W}$ and the $G_{F}$ coupling are defined by the $M_{W}$ parameter. The Higgs-boson mass constraint $m_{H}=125 \mathrm{GeV} / c^{2}$ ensures that the form-factor values remain in the vicinity of SM-fit values from LEP-1 and SLD [4,5].

The form factors are calculated in the massless-fermion approximation. Consequently, they only depend on the fermion weak isospin and charge, and are distinguished via three indices, $e$ (electron type), $u$ (up-quark type), and $d$ (down-quark type). For the $e e \rightarrow Z \rightarrow q \bar{q}$ process, the ZFITTER scattering-amplitude ansatz is

$$
\begin{aligned}
A_{q}= & \frac{i}{4} \frac{\sqrt{2} G_{F} M_{Z}^{2}}{\hat{s}-\left(M_{Z}^{2}-i \hat{s} \Gamma_{Z} / M_{Z}\right)} 4 T_{3}^{e} T_{3}^{q} \rho_{e q} \\
& \times\left[\left\langle\bar{e}\left|\gamma^{\mu}\left(1+\gamma_{5}\right)\right| e\right\rangle\left\langle\bar{q}\left|\gamma_{\mu}\left(1+\gamma_{5}\right)\right| q\right\rangle\right. \\
& -4\left|Q_{e}\right| \kappa_{e} \sin ^{2} \theta_{W}\left\langle\bar{e}\left|\gamma^{\mu}\right| e\right\rangle\left\langle\bar{q}\left|\gamma_{\mu}\left(1+\gamma_{5}\right)\right| q\right\rangle \\
& -4\left|Q_{q}\right| \kappa_{q} \sin ^{2} \theta_{W}\left\langle\bar{e}\left|\gamma^{\mu}\left(1+\gamma_{5}\right)\right| e\right\rangle\left\langle\bar{q}\left|\gamma_{\mu}\right| q\right\rangle \\
& \left.+16\left|Q_{e} Q_{q}\right| \kappa_{e q} \sin ^{4} \theta_{W}\left\langle\bar{e}\left|\gamma^{\mu}\right| e\right\rangle\left\langle\bar{q}\left|\gamma_{\mu}\right| q\right\rangle\right],
\end{aligned}
$$

where $q$ equals $u$ or $d$, the terms $\rho_{e q}, \kappa_{e}, \kappa_{q}$, and $\kappa_{e q}$ are complex-valued form factors, the bilinear $\gamma$ matrix terms are covariantly contracted, and $\frac{1}{2}\left(1+\gamma_{5}\right)$ is the left-handed helicity projector in the ZFITTER convention. The form factors are functions of the $\sin ^{2} \theta_{W}$ parameter and the Mandelstam $\hat{s}$ variable of the $e^{+} e^{-} \rightarrow Z \rightarrow f \bar{f}$ process. The $\kappa_{e}$ form factors of the $A_{u}$ and $A_{d}$ amplitudes are not equivalent; however, at $\hat{s}=M_{Z}^{2}$, they are numerically equal.

The $\rho_{e q}, \kappa_{e}$, and $\kappa_{q}$ form factors can be incorporated into QCD calculations as corrections to the Born-level $g_{A}^{f}$ and $g_{V}^{f}$ couplings,

$g_{V}^{f} \rightarrow \sqrt{\rho_{e q}}\left(T_{3}^{f}-2 Q_{f} \kappa_{f} \sin ^{2} \theta_{W}\right)$ and $g_{A}^{f} \rightarrow \sqrt{\rho_{e q}} T_{3}^{f}$,

where $f=e$ or $q$. The resulting current-current amplitude is similar to $A_{q}$, but the $\sin ^{4} \theta_{W}$ term contains $\kappa_{e} \kappa_{q}$. This difference is eliminated by adding the $\sin ^{4} \theta_{W}$ term of $A_{q}$ with the replacement of $\kappa_{e q}$ with $\kappa_{e q}-\kappa_{e} \kappa_{q}$ to the currentcurrent amplitude. Further details are in Ref. [7].

The products $\kappa_{f} \sin ^{2} \theta_{W}$, called effective mixing terms, are directly accessible from measurements of the asymmetry in the $\cos \vartheta$ distribution. However, neither the $\sin ^{2} \theta_{W}$ parameter nor the $\hat{s}$-dependent form factors can be inferred from measurements without assuming the SM. The effective mixing terms are denoted as $\sin ^{2} \theta_{\text {eff }}$ to distinguish them from the on-shell definition of the $\sin ^{2} \theta_{W}$ parameter, $\sin ^{2} \theta_{W}=1-M_{W}^{2} / M_{Z}^{2}$. The Drell-Yan process is most sensitive to the $\sin ^{2} \theta_{\text {eff }}$ mixing term of the lepton vertex, $\kappa_{e} \sin ^{2} \theta_{W}$. At the $Z$-boson pole, $\kappa_{e}$ is independent of the quark flavor, and the flavor-independent value of $\kappa_{e} \sin ^{2} \theta_{W}$ is commonly denoted as $\sin ^{2} \theta_{\text {eff }}^{\text {lept }}$. For comparisons with other measurements, the value of $\sin ^{2} \theta_{\text {eff }}^{\text {lept }}$ at the $Z$-boson pole is taken to be $\operatorname{Re}\left[\kappa_{e}\left(\sin ^{2} \theta_{W}, M_{Z}^{2}\right)\right] \sin ^{2} \theta_{W}$.
[1] S. D. Drell and T.-M. Yan, Massive Lepton Pair Production in Hadron-Hadron Collisions at High-Energies, Phys. Rev. Lett. 25, 316 (1970).

[2] J.C. Collins and D.E. Soper, Angular distribution of dileptons in high-energy hadron collisions, Phys. Rev. D 16, 2219 (1977).

[3] C. Patrignani et al. (Particle Data Group), Review of particle physics, Chin. Phys. C 40, 100001 (2016).

[4] The measurements are $A_{\mathrm{fb}}^{0, \ell}, \mathcal{A}_{\ell}\left(P_{\tau}\right), \mathcal{A}_{\ell}(\mathrm{SLD}), A_{\mathrm{fb}}^{0, \mathrm{~b}}, A_{\mathrm{fb}}^{0, \mathrm{c}}$, and $Q_{\mathrm{fb}}^{\text {had }}$ in The ALEPH, DELPHI, L3, OPAL, SLD Collaborations, the LEP Electroweak Working Group, the SLD Electroweak and Heavy Flavour Groups, Precision electroweak measurements on the $Z$ resonance, Phys. Rep. 427, 257 (2006).

[5] The ALEPH, DELPHI, L3, OPAL Collaborations, the LEP Electroweak Working Group, Electroweak Measurements in
Electron-Positron Collisions at W-Boson-Pair Energies at LEP, Phys. Rep. 532, 119 (2013).

[6] D. Acosta et al. (CDF Collaboration), Measurement of the forward-backward charge asymmetry of electron positron pairs in $p \bar{p}$ collisions at $\sqrt{s}=1.96 \mathrm{TeV}$, Phys. Rev. D 71, 052002 (2005).

[7] T. Aaltonen et al. (CDF Collaboration), Indirect measurement of $\sin ^{2} \theta_{W}\left(M_{W}\right)$ using $e^{+} e^{-}$pairs in the Z-boson region with $p \bar{p}$ collisions at a center-of-momentum energy of $1.96 \mathrm{TeV}$, Phys. Rev. D 88, 072002 (2013); Erratum, Phys. Rev. D 88, 079905 (2013).

[8] T. Aaltonen et al. (CDF Collaboration), Indirect measurement of $\sin ^{2} \theta_{W}$ (or $M_{W}$ ) using $\mu^{+} \mu^{-}$pairs from $\gamma^{*} / Z$ bosons produced in $p \bar{p}$ collisions at a center-of-momentum energy of $1.96 \mathrm{TeV}$, Phys. Rev. D 89, 072005 (2014). 
[9] T. Aaltonen et al. (CDF Collaboration), Measurement of $\sin ^{2} \theta_{\text {eff }}^{\text {lept }}$ using $e^{+} e^{-}$pairs from $\gamma^{*} / Z$ bosons produced in $p \bar{p}$ collisions at a center-of-momentum energy of $1.96 \mathrm{TeV}$, Phys. Rev. D 93, 112016 (2016).

[10] V. M. Abazov et al. (D0 Collaboration), Measurement of the Forward-Backward Charge Asymmetry and Extraction of $\sin ^{2} \theta_{W}^{\text {eff }}$ in $p \bar{p} \rightarrow Z / \gamma^{*}+X \rightarrow e^{+} e^{-}+X$ Events Produced at $\sqrt{s}=1.96 \mathrm{TeV}$, Phys. Rev. Lett. 101, 191801 (2008).

[11] V. M. Abazov et al. (D0 Collaboration), Measurement of $\sin ^{2} \theta_{\text {eff }}^{\ell}$ and Z-light quark couplings using the forwardbackward charge asymmetry in $p \bar{p} \rightarrow Z / \gamma^{*} \rightarrow e^{+} e^{-}$events with $\mathcal{L}=5.0 \mathrm{fb}^{-1}$ at $\sqrt{s}=1.96 \mathrm{TeV}$, Phys. Rev. D 84, 012007 (2011).

[12] V. M. Abazov et al. (D0 Collaboration), Measurement of the Effective Weak Mixing Angle in $p \bar{p} \rightarrow Z / \gamma^{*} \rightarrow e^{+} e^{-}$ Events, Phys. Rev. Lett. 115, 041801 (2015).

[13] V. M. Abazov et al. (D0 Collaboration), Measurement of the Effective Weak Mixing Angle in $p \bar{p} \rightarrow Z / \gamma^{*} \rightarrow l^{+} l^{-}$ Events, arXiv:1710.03951 [Phys. Rev. Lett. (to be published)].

[14] G. Aad et al. (ATLAS Collaboration), Measurement of the forward-backward asymmetry of electron and muon pairproduction in $p p$ collisions at $\sqrt{s}=7 \mathrm{TeV}$ with the ATLAS detector, J. High Energy Phys. 09 (2015) 049.

[15] S. Chatrchyan et al. (CMS Collaboration), Measurement of the weak mixing angle with the Drell-Yan process in protonproton collisions at the LHC, Phys. Rev. D 84, 112002 (2011).

[16] R. Aaij et al. (LHCb Collaboration), Measurement of the forward-backward asymmetry in $Z / \gamma^{*} \rightarrow \mu^{+} \mu^{-}$decays and determination of the effective weak mixing angle, J. High Energy Phys. 11 (2015) 190.

[17] A. Sirlin, Radiative corrections in the $\mathrm{SU}(2)_{L} \times \mathrm{U}(1)$ theory: A simple renormalization framework, Phys. Rev. D 22, 971 (1980).

[18] E. Mirkes, Angular decay distribution of leptons from W bosons at NLO in hadronic collisions, Nucl. Phys. B387, 3 (1992).

[19] E. Mirkes and J. Ohnemus, $W$ and $Z$ polarization effects in hadronic collisions, Phys. Rev. D 50, 5692 (1994).

[20] D. Bardin, M. Bilenky, T. Riemann, M. Sachwitz, and H. Vogt, Dizet: Electroweak one-loop corrections for $e^{+}+$ $e^{-} \rightarrow f^{+}+f^{-}$around the $Z^{0}$ peak, Comput. Phys. Commun. 59, 303 (1990).

[21] D. Bardin, P. Christova, M. Jack, L. Kalinovskaya, A. Olchevski, S. Riemann, and T. Riemann, ZFITTER v.6.21: A semi-analytical program for fermion pair production in $e^{+} e^{-}$annihilation, Comput. Phys. Commun. 133, 229 (2001).

[22] A. Arbuzov, M. Awramik, M. Czakon, A. Freitas, M. Grünewald, K. Monig, S. Riemann, and T. Riemann, ZFITTER: a semi-analytical program for fermion pair production in $e^{+} e^{-}$annihilation, from version 6.21 to version 6.42, Comput. Phys. Commun. 174, 728 (2006).

[23] R. D. Ball et al. (NNPDF Collaboration), Parton distributions for the LHC Run II, J. High Energy Phys. 04 (2015) 040 .

[24] R. D. Ball et al. (NNPDF Collaboration), Parton distributions with LHC data, Nucl. Phys. B867, 244 (2013).

[25] R. D. Ball, V. Bertone, F. Cerutti, L. Del Debbio, S. Forte, A. Guffanti, J. I. Latorre, J. Rojo, and M. Ubiali (NNPDF
Collaboration), Unbiased global determination of parton distributions and their uncertainties at NNLO and at LO, Nucl. Phys. B855, 153 (2012).

[26] R. D. Ball, V. Bertone, F. Cerutti, L. Del Debbio, S. Forte, A. Guffanti, J. I. Latorre, J. Rojo, and M. Ubiali (NNPDF Collaboration), Impact of heavy quark masses on parton distributions and LHC phenomenology, Nucl. Phys. B849, 296 (2011).

[27] R. D. Ball, L. Del Debbio, S. Forte, A. Guffanti, J. I. Latorre, J. Rojo, and M. Ubiali (NNPDF Collaboration), A first unbiased global NLO determination of parton distributions and their uncertainties, Nucl. Phys. B838, 136 (2010).

[28] R. D. Ball, L. Del Debbio, S. Forte, A. Guffanti, J. I. Latorre, A. Piccione, J. Rojo, and M. Ubiali (NNPDF Collaboration), A determination of parton distributions with faithful uncertainty estimation, Nucl. Phys. B809, 1 (2009); Erratum, Nucl. Phys. B816, 293 (2009).

[29] S. Forte, L. Garrido, J. I. Latorre, and A. Piccione, Neural network parametrization of deep inelastic structure functions, J. High Energy Phys. 05 (2002) 062.

[30] A. Abulencia et al. (CDF Collaboration), Measurements of inclusive $W$ and $Z$ cross sections in $p \bar{p}$ collisions at $\sqrt{s}=1.96 \mathrm{TeV}$, J. Phys. G 34, 2457 (2007); T. Affolder et al., CDF central outer tracker, Nucl. Instrum. Methods Phys. Res., Sect. A 526, 249 (2004); T. Aaltonen et al., Operational experience, improvements, and performance of the CDF Run II Silicon Vertex Detector, Nucl. Instrum. Methods Phys. Res., Sect. A 729, 153 (2013); L. Balka et al., The CDF central electromagnetic calorimeter, Nucl. Instrum. Methods Phys. Res., Sect. A 267, 272 (1988); S. Bertolucci et al., The CDF central and endwall hadron calorimeter, Nucl. Instrum. Methods Phys. Res., Sect. A 267, 301 (1988); M. Albrow et al., The CDF plug upgrade electromagnetic calorimeter: Test beam results, Nucl. Instrum. Methods Phys. Res., Sect. A 480, 524 (2002); P. de Barbaro, CDF end plug hadron calorimeter upgrade: Design, production and quality control results, IEEE Trans. Nucl. Sci. 42, 510 (1995); G. Apollinari, K. Goulianos, P. Melese, and M. Lindgren, Shower maximum detector for the CDF plug upgrade calorimeter, Nucl. Instrum. Methods Phys. Res., Sect. A 412, 515 (1998); G. Ascoli, L. E. Holloway, I. Karliner, U. E. Kruse, R. D. Sard, V. J. Simaitis, D. A. Smith, and T. K. Westhusing, CDF central muon detector, Nucl. Instrum. Methods Phys. Res., Sect. A 268, 33 (1988); D. Acosta et al., The performance of the CDF luminosity monitor, Nucl. Instrum. Methods Phys. Res., Sect. A 494, 57 (2002); E. J. Thomson et al., Online track processor for the CDF upgrade, IEEE Trans. Nucl. Sci. 49, 1063 (2002); W. Ashmanskas et al., The CDF silicon vertex trigger, Nucl. Instrum. Methods Phys. Res., Sect. A 518, 532 (2004); L. Ristori and G. Punzi, Triggering on heavy flavors at hadron colliders, Annu. Rev. Nucl. Part. Sci. 60, 595 (2010); A. Adelman et al., The Silicon vertex trigger upgrade at CDF, Nucl. Instrum. Methods Phys. Res., Sect. A 572, 361 (2007).

[31] S. Abachi et al. (D0 Collaboration), The D0 Detector, Nucl. Instrum. Methods Phys. Res., Sect. A 338, 185 (1994); M. Fortner, A. Maciel, H. Evans, B. Kothari, and S. Uzunyan, The level-2 muon trigger at D0, IEEE Trans. Nucl. Sci. 49, 1589 (2002); M. Abolins et al., The run IIb trigger upgrade 
for the D0 experiment, IEEE Trans. Nucl. Sci. 51, 340 (2004); R. D. Angstadt et al., The DZERO level 3 data acquisition system, IEEE Trans. Nucl. Sci. 51, 445 (2004); V. M. Abazov et al., The Muon system of the run II D0 detector, Nucl. Instrum. Methods Phys. Res., Sect. A 552, 372 (2005); The upgraded D0 detector, Nucl. Instrum. Methods Phys. Res., Sect. A 565, 463 (2006); M. Abolins et al., Design and implementation of the new D0 level-1 calorimeter trigger, Nucl. Instrum. Methods Phys. Res., Sect. A 584, 75 (2008); R. Angstadt et al., The layer 0 inner silicon detector of the D0 experiment, Nucl. Instrum. Methods Phys. Res., Sect. A 622, 298 (2010); S. N. Ahmed et al., The D0 silicon microstrip tracker, Nucl. Instrum. Methods Phys. Res., Sect. A 634, 8 (2011); V. M. Abazov et al. (D0 Collaboration), Muon reconstruction and identification with the Run II D0 detector, Nucl. Instrum. Methods Phys. Res., Sect. A 737, 281 (2014); Electron and photon identification in the D0 experiment, Nucl. Instrum. Methods Phys. Res., Sect. A 750, 78 (2014).

[32] A. Bodek, A simple event weighting technique for optimizing the measurement of the forward-backward asymmetry of Drell-Yan dilepton pairs at hadron colliders, Eur. Phys. J. C 67, 321 (2010).

[33] C. Hays, Y. Huang, A. V. Kotwal, H. Gerberich, S. Menzemer, K. Rinnert, C. Lecci, M. Herndon, and F. D. Snider, Inside-out tracking at CDF, Nucl. Instrum. Methods Phys. Res., Sect. A 538, 249 (2005).

[34] T. Sjöstrand, P. Edén, L. Lönnblad, G. Miu, S. Mrenna, and E. Norrbin, High-energy physics event generation with PYTHIA 6.1, Comput. Phys. Commun. 135, 238 (2001).

[35] H. L. Lai, J. Huston, S. Kuhlmann, J. Morfin, F. Olness, J. F. Owens, J. Pumplin, and W. K. Tung (CTEQ Collaboration), Global QCD analysis of parton structure of the nucleon: CTEQ5 parton distributions, Eur. Phys. J. C 12, 375 (2000).

[36] E. Barberio and Z. Was, PHOTOS: A Universal Monte Carlo for QED radiative corrections. Version 2.0, Comput. Phys. Commun. 79, 291 (1994).

[37] E. Barberio, B. van Eijk, and Z. Was, PHOTOS: A Universal Monte Carlo for QED radiative corrections in decays, Comput. Phys. Commun. 66, 115 (1991).

[38] P. Golonka and Z. Was, PHOTOS Monte Carlo: A Precision tool for QED corrections in $Z$ and $W$ decays, Eur. Phys. J. C 45, 97 (2006).

[39] G. Grindhammer, M. Rudowicz, and S. Peters, The fast simulation of electromagnetic and hadronic showers, Nucl. Instrum. Methods Phys. Res., Sect. A 290, 469 (1990).

[40] G. A. Ladinsky and C.-P. Yuan, Nonperturbative regime in QCD resummation for gauge boson production at hadron colliders, Phys. Rev. D 50, R4239 (1994).

[41] C. Balàzs and C.-P. Yuan, Soft gluon effects on lepton pairs at hadron colliders, Phys. Rev. D 56, 5558 (1997).

[42] F. Landry, R. Brock, P. M. Nadolsky, and C.-P. Yuan, Tevatron Run-1 Z boson data and Collins-Soper-Sterman resummation formalism, Phys. Rev. D 67, 073016 (2003).

[43] A. Konychev and P. Nadolsky, Universality of the CollinsSoper-Sterman nonperturbative function in gauge boson production, Phys. Lett. B 633, 710 (2006).
[44] P. M. Nadolsky, H.-L. Lai, Q.-H. Cao, J. Huston, J. Pumplin, D. Stump, W.-K. Tung, and C.-P. Yuan (CTEQ Collaboration), Implications of CTEQ global analysis for collider observables, Phys. Rev. D 78, 013004 (2008).

[45] T. Sjöstrand, S. Mrenna, and P. Z. Skands, PYTHIA 6.4 Physics and Manual, J. High Energy Phys. 05 (2006) 026.

[46] A. Bodek, A. van Dyne, J.-Y. Han, W. Sakumoto, and A. Strelnikov, Extracting muon momentum scale corrections for hadron collider experiments, Eur. Phys. J. C 72, 2194 (2012).

[47] S. Frixione, P. Nason, and C. Oleari, Matching NLO QCD computations with parton shower simulations: The POWHEG method, J. High Energy Phys. 11 (2007) 070.

[48] S. Alioli, P. Nason, C. Oleari, and E. Re, NLO vector-boson production matched with shower in POWHEG, J. High Energy Phys. 07 (2008) 060.

[49] N. Sato, J. F. Owens, and H. Prosper, Bayesian reweighting for global fits, Phys. Rev. D 89, 114020 (2014).

[50] W. T. Giele and S. Keller, Implications of hadron collider observables on parton distribution function uncertainties, Phys. Rev. D 58, 094023 (1998).

[51] A. Bodek, J.-Y. Han, A. Khukhunaishvili, and W. Sakumoto, Using Drell-Yan forward-backward asymmetry to reduce PDF uncertainties in the measurement of electroweak parameters, Eur. Phys. J. C 76, 115 (2016).

[52] H.-L. Lai, M. Guzzi, J. Huston, Z. Li, P. Nadolsky, J. Pumplin, and C.-P. Yuan, New parton distributions for collider physics, Phys. Rev. D 82, 074024 (2010).

[53] R. Brun and F. Carminati, CERN Program Library Long Writeup W5013, 1993 (unpublished).

[54] M. L. Mangano, F. Piccinini, A. D. Polosa, M. Moretti, and R. Pittau, ALPGEN, a generator for hard multiparton processes in hadronic collisions, J. High Energy Phys. 07 (2003) 001.

[55] R. Hamberg, W. L. van Neerven, and T. Matsuura, A complete calculation of the order $\alpha_{s}^{2}$ correction to the Drell-Yan $K$ factor, Nucl. Phys. B359, 343 (1991); Erratum, Nucl. Phys. B644, 403 (2002).

[56] L. Lyons, D. Gibaut, and P. Cliffort, How to combine correlated estimates of a single physical quantity, Nucl. Instrum. Methods Phys. Res., Sect. A 270, 110 (1988).

[57] T. Aaltonen et al. (CDF and D0 Collaborations), Combination of the top-quark mass measurements from the Tevatron collider, Phys. Rev. D 86, 092003 (2012).

[58] T. Aaltonen et al. (CDF and D0 Collaboration), Combination of CDF and D0 $W$-boson mass measurements, Phys. Rev. D 88, 052018 (2013).

[59] S. Bethke, The 2009 world average of $\alpha_{s}$, Eur. Phys. J. C 64, 689 (2009).

[60] F. Jegerlehner, Electroweak effective couplings for future precision experiments, Nuovo Cimento Soc. Ital. Fis. 034S1C, 31 (2011). 